The excited-state structure, vibrations, lifetimes, and nonradiative dynamics of jetcooled 1-methylcytosine

Maria A. Trachsel, Timo Wiedmer, Susan Blaser, Hans-Martin Frey, Quansong Li, Sergi Ruiz-Barragan, Lluís Blancafort', and Samuel Leutwyler'

Citation: J. Chem. Phys. 145, 134307 (2016); doi: 10.1063/1.4964091

View online: http://dx.doi.org/10.1063/1.4964091

View Table of Contents: http://aip.scitation.org/toc/jcp/145/13

Published by the American Institute of Physics

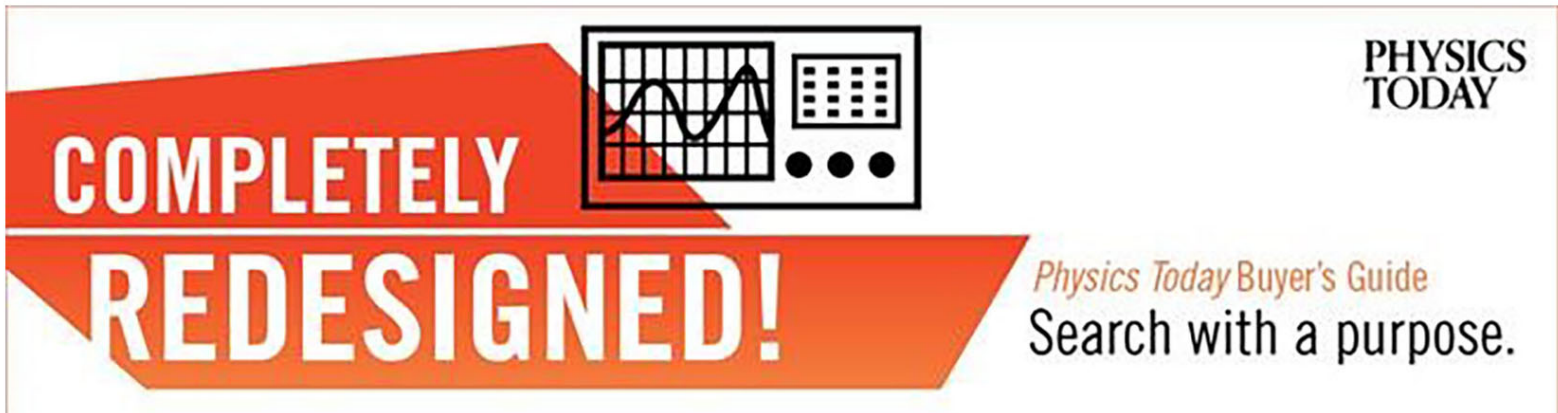




\title{
The excited-state structure, vibrations, lifetimes, and nonradiative dynamics of jet-cooled 1-methylcytosine
}

\author{
Maria A. Trachsel, ${ }^{1}$ Timo Wiedmer, ${ }^{1}$ Susan Blaser, ${ }^{1}$ Hans-Martin Frey, ${ }^{1}$ Quansong Li, ${ }^{2}$ \\ Sergi Ruiz-Barragan, ${ }^{3}$ Lluís Blancafort, ${ }^{4, a)}$ and Samuel Leutwyler ${ }^{1, b)}$ \\ ${ }^{1}$ Department of Chemistry and Biochemistry, University of Bern, Freiestrasse 3, CH-3012 Bern, Switzerland \\ ${ }^{2}$ Key Laboratory of Cluster Science of Ministry of Education, School of Chemistry, Beijing Institute \\ of Technology, 100081 Beijing, China \\ ${ }^{3}$ Lehrstuhl für Theoretische Chemie, Ruhr-Universitat Bochum, 44780 Bochum, Germany \\ ${ }^{4}$ Institut de Química Computacional i Catàlisi and Departament de Química, Universitat de Girona, \\ Facultat de Ciències, C/ M. Aurèlia Campmany 69, 17003 Girona, Spain
}

(Received 15 July 2016; accepted 21 September 2016; published online 7 October 2016)

\begin{abstract}
We have investigated the $S_{0} \rightarrow S_{1}$ UV vibronic spectrum and time-resolved $S_{1}$ state dynamics of jet-cooled amino-keto 1-methylcytosine (1MCyt) using two-color resonant two-photon ionization, UV/UV holeburning and depletion spectroscopies, as well as nanosecond and picosecond timeresolved pump/delayed ionization measurements. The experimental study is complemented with spin-component-scaled second-order coupled-cluster and multistate complete active space second order perturbation ab initio calculations. Above the weak electronic origin of $1 \mathrm{MCyt}$ at $31852 \mathrm{~cm}^{-1}$ about 20 intense vibronic bands are observed. These are interpreted as methyl group torsional transitions coupled to out-of-plane ring vibrations, in agreement with the methyl group rotation and out-of-plane distortions upon ${ }^{1} \pi \pi^{*}$ excitation predicted by the calculations. The methyl torsion and $v_{1}^{\prime}$ (butterfly) vibrations are strongly coupled, in the $S_{1}$ state. The $S_{0} \rightarrow S_{1}$ vibronic spectrum breaks off at a vibrational excess energy $E_{\text {exc }} \sim 500 \mathrm{~cm}^{-1}$, indicating that a barrier in front of the ethylene-type $S_{1} \leadsto S_{0}$ conical intersection is exceeded, which is calculated to lie at $E_{\text {exc }}=366 \mathrm{~cm}^{-1}$. The $S_{1} \leadsto S_{0}$ internal conversion rate constant increases from $k_{I C}=2 \cdot 10^{9} \mathrm{~s}^{-1}$ near the $S_{1}(v=0)$ level to $1 \cdot 10^{11} \mathrm{~s}^{-1}$ at $E_{\text {exc }}=516 \mathrm{~cm}^{-1}$. The ${ }^{1} \pi \pi^{*}$ state of $1 \mathrm{MCyt}$ also relaxes into the lower-lying triplet $T_{1}\left({ }^{3} \pi \pi^{*}\right)$ state by intersystem crossing (ISC); the calculated spin-orbit coupling (SOC) value is $2.4 \mathrm{~cm}^{-1}$. The ISC rate constant is 10-100 times lower than $k_{I C}$; it increases from $k_{I S C}=2 \cdot 10^{8} \mathrm{~s}^{-1}$ near $S_{1}(v=0)$ to $k_{I S C}=2 \cdot 10^{9} \mathrm{~s}^{-1}$ at $E_{\text {exc }}=516 \mathrm{~cm}^{-1}$. The $T_{1}$ state energy is determined from the onset of the time-delayed photoionization efficiency curve as $25600 \pm 500 \mathrm{~cm}^{-1}$. The $T_{2}\left({ }^{3} n \pi^{*}\right)$ state lies $>1500 \mathrm{~cm}^{-1}$ above $S_{1}(v=0)$, so $S_{1} \leadsto T_{2}$ ISC cannot occur, despite the large SOC parameter of $10.6 \mathrm{~cm}^{-1}$. An upper limit to the adiabatic ionization energy of 1MCyt is determined as $8.41 \pm 0.02 \mathrm{eV}$. Compared to cytosine, methyl substitution at $\mathrm{N} 1$ lowers the adiabatic ionization energy by $\geq 0.32 \mathrm{eV}$ and leads to a much higher density of vibronic bands in the $S_{0} \rightarrow S_{1}$ spectrum. The effect of methylation on the radiationless decay to $S_{0}$ and ISC to $T_{1}$ is small, as shown by the similar break-off of the spectrum and the similar computed mechanisms. Published by AIP Publishing. [http://dx.doi.org/10.1063/1.4964091]
\end{abstract}

\section{INTRODUCTION}

In DNA, cytosine (Cyt) occurs in the form of 2'deoxycytidine, in which deoxyribose is bound to the Cyt $\mathrm{N} 1$ atom. In the gas phase, however, the most stable forms of unsubstituted cytosine are the cis- and trans-enol-amino tautomers. ${ }^{1-8}$ In 1-methylcytosine (1MCyt) the substitution of a methyl group at N1 eliminates these enol-amino forms and renders the amino-keto tautomer 1, see Figure 1, as the most stable form, thereby providing a better mimic for cytidine. De Vries and co-workers have measured the resonant two-photon ionization (R2PI) spectrum of 1MCyt with an electronic origin at $31908 \mathrm{~cm}^{-1}$ followed by several vibronic bands and assigned it to the $S_{0} \rightarrow S_{1}$ transition of the amino-keto tautomer $\mathbf{1}$, $^{9,10}$

\footnotetext{
a)E-mail: lluis.blancafort@udg.edu

b) E-mail: leutwyler@dcb.unibe.ch
}

The photophysics of Cyt and its derivatives in the gas phase has been widely studied. When using fs pump/ionization techniques with excitation at 250-267 nm, excited-state transients have been measured that exhibit mono-, bi-, or tri-exponential lifetimes ranging from $\tau=50$ fs to $3.8 \mathrm{ps,}$ depending on the experimental setup. ${ }^{11-14}$ For Cyt, slower decay components of $\tau=19 \mathrm{ps}$ and $55 \mathrm{ps}$ have been also observed after excitation at longer wavelengths of 270 and $280 \mathrm{~nm}$, respectively. ${ }^{13,14}$ In the red wing of the spectrum, excitation at $290 \mathrm{~nm}$ gives rise to a component with a lifetime of $\geq 150$ ps. $^{13}$ Lobsiger et al. determined a lower limit to the lifetime of the Cyt $S_{1} ; v=0$ level as $\tau=45$ ps by modeling the Lorentzian broadening of the rovibronic band contours in the R2PI spectrum. ${ }^{8}$ Using ps pump/ionization techniques, Blaser et al. have recently measured the lifetime at the Cyt $S_{1} 0_{0}^{0}$ band as $\tau=730 \mathrm{ps}{ }^{15}$ In the R2PI spectra, the short lifetimes are reflected in a break-off of the sharp vibronic 


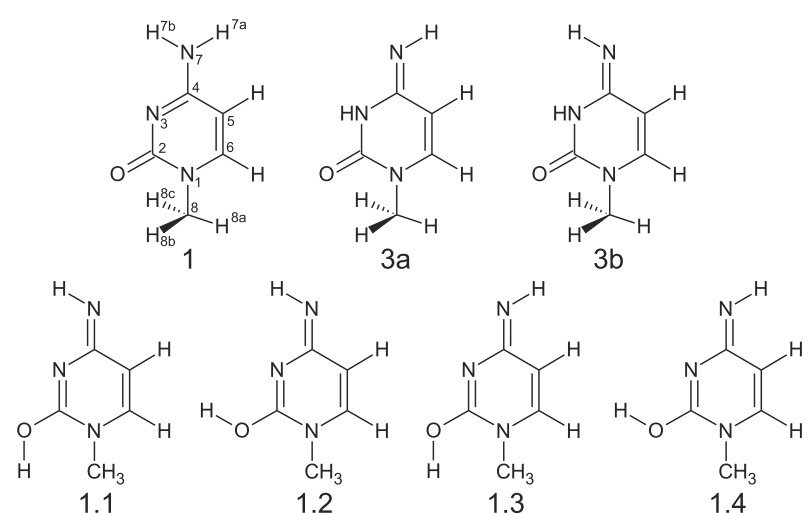

FIG. 1. The seven tautomers and rotamers of 1MCyt.

bands at vibrational excess energies $E_{\text {exc }} \sim 500 \mathrm{~cm}^{-1}$ above the $0_{0}^{0}$ transition. ${ }^{8-10}$

When exciting Cyt at its $0_{0}^{0}$ band $\left(313 \mathrm{~nm}, 31900 \mathrm{~cm}^{-1}\right)$ followed by ionization at $157 \mathrm{~nm}$, an excited-state signal with a lifetime of $\sim 270 \mathrm{~ns}$ is also observed; this signal has been attributed to a triplet state. ${ }^{9,10}$ Via the measurement of the singlet and triplet state ionization energies (IEs), this state has been identified as the $T_{1}$ state. ${ }^{16}$ More recently, the intersystem crossing (ISC) quantum yield and ISC rates of Cyt have been measured as a function of the $S_{1}$ state vibrational energy. ${ }^{17}$ A long-lived excited state with a lifetime of several hundred ns has also been observed for 1MCyt following excitation near the $0_{0}^{0}$ band and ionization at $157 \mathrm{~nm}^{9}$

The interest in the photophysics of Cyt has motivated many theoretical studies, whose main aims were to explain the ultrashort excited-state lifetimes and the formation of the long-lived triplet state. ${ }^{18-34}$ Most studies have considered the amino-keto tautomer. Among the points of debate is whether the structure of the $S_{1}$ minimum of $\left(\pi \pi^{*}\right)$ character, ${ }^{1}(\pi \pi)^{*}{ }_{\text {Min }}$, has a quasi-planar or ring-puckered conformation, for a recent discussion see Ref. 34. The ultrafast decay to the ground state has been explained by the presence of conical intersections (CI) between the singlet ground and first excited states. Three different CI structures are accessible, depending on the experimental excitation energies: The lowest-energy one, called $(E t h)_{X}$, is characterized by an out-of-plane twist of the $\mathrm{C}_{5}-\mathrm{C}_{6}$ double bond, similar to the CI structure of ethylene. ${ }^{21-26,30-32}$ The second CI, called $(O P)_{X}$, has a puckered $\mathrm{N}_{3}$ atom and the amino group bent out of the plane. ${ }^{18,24-26,30,32}$ The third structure, $\left(n_{O} \pi^{*}\right)_{X}$, has a semi-planar ring and a stretched $\mathrm{C}_{2}-\mathrm{O}_{7}$ bond. ${ }^{18,24-26,30,32}$ The predicted decay mechanism of Cyt depends strongly on the level of theory, as discussed extensively in the literature. With methods that include dynamic correlation, such as complete active space second order perturbation (CASPT2), multi-reference configuration interaction (MRCI) and density functional theory (DFT) combined with MRCI (DFT/MRCI), the energetically favored path is associated with $(E t h)_{X}$. It goes through a minimum on $S_{1},{ }^{1}(\pi \pi)^{*}{ }_{\operatorname{Min}}$, and leads to the intersection through a barrier. The estimated barrier heights are $900 \mathrm{~cm}^{-1}$ with $\mathrm{MRCI}^{25}$ and CASPT2//complete active space self-consistent field (CASSCF) (CASPT2 energies calculated along a CASSCF optimized path), ${ }^{24}$ and $645 \mathrm{~cm}^{-1}$ at the multistate CASPT2 (MS-CASPT2) level (geometries optimized at the same level of theory). ${ }^{32}$ The barrier to access $(O P)_{X}$ from $^{1}(\pi, \pi)^{*}{ }_{\text {Min }}$ is $1050 \mathrm{~cm}^{-1}$ at the MRCI level, ${ }^{25} 970 \mathrm{~cm}^{-1}$ at the CASPT2//CASSCF level, ${ }^{24}$ and $890 \mathrm{~cm}^{-1}$ with MS-CASPT2. ${ }^{32}$ The energy of $\left(n_{O} \pi^{*}\right)_{X}$ at the CASPT2//CASSCF and MS-CASPT2 levels of theory is more than $8400 \mathrm{~cm}^{-1}$ above ${ }^{1}(\pi, \pi)^{*}{ }_{\text {Min. }}{ }^{24,32}$ This corresponds to excitation at $248 \mathrm{~nm}$ and suggests that the $\left(n_{O} \pi^{*}\right)_{X}$ intersection is not relevant for any of the experiments related above. In 1MCyt, upper bounds of $\sim 3100$ and $\sim 5200 \mathrm{~cm}^{-1}$ for the barriers to access $(E t h)_{X}$ and $(O P)_{X}$ have been provided at the MS-CASPT2//CASSCF level. ${ }^{35}$ Recently, the MS-CASPT2 barrier height for the decay through $(E t h)_{X}$ was estimated as $1600 \mathrm{~cm}^{-1} .36$

An early study on triplet formation in Cyt indicated the occurrence of competitive intersystem crossing (ISC) at a region of high spin-orbit coupling (SOC) along the decay path to the $\left(n_{O} \pi^{*}\right)_{X}$ state. ${ }^{37}$ However, that study only gives a partial account of the possible mechanisms since the ethylenic and out-of-plane mechanisms were not considered at that time. Intersystem crossing on a time scale competitive with the radiationless decay was observed in a trajectory surface hopping (TSH) dynamics study carried out at the CASSCF level. ${ }^{38}$ More recently, $S_{1} \rightarrow T_{1}$ ISC has been suggested as the mechanism for formation of the triplet after excitation near the origin. ${ }^{17}$

In this context, our joint experimental and theoretical study combining R2PI and UV/UV holeburning spectroscopy of 1MCyt with high level $a b$ initio calculations addresses several goals: (1) To interpret and explain the vibronic structure of the $S_{0} \rightarrow S_{1}$ R2PI spectrum, which depends on the change between the $S_{0^{-}}$and $S_{1}$-state structures and the excited-state vibrations that are activated by the optical excitation. In order to check the possible involvement of the methyl group rotation, we have also measured the isotopomer 1-( $\left.d_{3}\right)$-methylcytosine [1-(d $\left.d_{3}\right)$-MCyt]. (2) To investigate the decay mechanism of 1-MCyt and 1-( $\left.d_{3}\right)$-MCyt that leads to the break-off of the vibronic structure about $500 \mathrm{~cm}^{-1}$ above the $0_{0}^{0}$ band of the $S_{0} \rightarrow S_{1}$ spectrum, to assign a decay path with MS-CASPT2//CASSCF calculations, and to measure the internal conversion (IC) rates $k_{I C}$ as a function of $S_{1}$ state excess vibrational energy $E_{\text {exc }}$. (3) To confirm the formation of a long-lived triplet state measured by R2PI at long delays, $, 9,10$ to study the intersystem crossing (ISC) rate constants as a function of $E_{\text {exc }}$, and determine its mechanism of formation.

\section{COMPUTATIONAL AND EXPERIMENTAL METHODS}

\section{A. Theoretical methods}

A uniform theoretical treatment of the ground- and excited-state potential energy surfaces of 1MCyt is difficult, and we have combined several theoretical approaches. The ground-state calculations have been carried out at the B3LYP, spin-component-scaled (SCS) resolution-of-identity (RI) Møller-Plesset (MP2) and SCS approximate secondorder coupled-cluster (CC2) levels of theory with the TZVP, aug-cc-pVTZ and aug-cc-pVDZ basis sets, respectively. The 
excited-state calculations in the vicinity of the Franck-Condon (FC) region (vertical spectrum and excited-state minima) have been done at the time dependent (TD) CAM-B3LYP/6-311G* and SCS-CC2/aug-cc-pVDZ levels of theory. Finally, the CI optimization and the calculation of the reaction paths have been carried out with $\mathrm{CASSCF} / 6-311 \mathrm{G}^{* *}$ because of the multi-referential character of the wave function in these regions. To provide a uniform global description, the final excited-state energies are obtained from MS-CASPT2 singlepoint calculations on the optimized structures using the atomic natural orbital (ANO)-L basis contracted to $4 \mathrm{~s} 3 \mathrm{p} 2 \mathrm{~d}$ for $\mathrm{C}, \mathrm{N}$, and $\mathrm{O}$ and $3 \mathrm{~s} 2 \mathrm{p} 1 \mathrm{~d}$ for $\mathrm{H}$. For the CASPT2 calculations, we have used an imaginary level shift ${ }^{39}$ of 0.1 a.u. and the standard ionization potential-electron affinity correction ${ }^{40}$ of 0.25 a.u.

The active space for the MS-CASPT2/ANO-L calculations at the FC region is $(14,10)(14$ electrons in 10 orbitals) and includes the $8 \pi$ orbitals and the two lone pairs on the oxygen and nitrogen atoms. The underlying wave function has been averaged over 8 states with equal weights, and all states are included in the MS-CASPT2 treatment. The active space of the CASSCF calculations of the excited-state decay paths and the corresponding MSCASPT2 single-point calculations was specifically tailored for each path (see the supplementary material for details). This procedure yields the CI structures with small MS-CASPT2 $S_{1} / S_{0}$ energy gaps of $887 \mathrm{~cm}^{-1}(0.11 \mathrm{eV})$ at $(\text { Eth })_{X}$ and $2820 \mathrm{~cm}^{-1}(0.35 \mathrm{eV})$ at $(O P)_{X}$. To calculate the reaction paths we have optimized the transition structures (TS) on $S_{1}$, and the paths were obtained combining the intrinsic reaction coordinate $^{41}$ and initial relaxation direction ${ }^{42}$ techniques. The calculated barriers also include vibrational zero-point energy (ZPE) corrections, based on CASSCF frequencies at $\left({ }^{1} \pi \pi^{*}\right)_{\text {Min }}$ (with $3 N-6$ vibrational modes) and at the corresponding TS (including $3 N-7$ modes). The CI were optimized using the recently developed double NewtonRaphson algorithm. ${ }^{43}$ The calculations were performed using Turbomole $6.4,{ }^{44,45}$ a modified version of Gaussian $09^{46}$ and Molcas 7.8. ${ }^{47,48}$

\section{B. Experimental methods}

$1 \mathrm{MCyt}$ and $1-d_{3}$-MCyt were synthesized as described in the literature. ${ }^{49}$ The molecular-beam/mass spectrometer experimental setup has been previously described. ${ }^{50}$ Neon carrier gas at 1.8 bar backing pressure is passed through a $0.4 \mathrm{~mm}$ diam. pulsed nozzle containing $1 \mathrm{MCyt}$ or $1-d_{3}$-MCyt heated to $220^{\circ} \mathrm{C}$. Mass-selected two-color resonant two-photon ionization (2C-R2PI) spectra over the $31200-33000 \mathrm{~cm}^{-1}$ range are measured by crossing the skimmed supersonic jet with unfocused UV excitation and ionization laser beams in the source of a linear time-offlight mass spectrometer. Excitation is performed with the frequency-doubled output of a NarrowScan D-R dye laser $(0.4-0.6 \mathrm{~mJ} / \mathrm{pulse})$, which is reduced to $0.3 \mathrm{~mJ} / \mathrm{pulse}$ for the rotational contour measurements. The laser bandwidth in the $\mathrm{UV}$ is $0.053 \mathrm{~cm}^{-1}(1600 \mathrm{MHz})$ full-width at half-maximum (FWHM). The UV ionization pulses at $223 \mathrm{~nm}$ are generated by an Ekspla NT342B UV optical parametric oscillator
(OPO) $\left(1.5 \mathrm{~mJ} /\right.$ pulse, $5 \mathrm{~cm}^{-1}$ bandwidth). Photoionization efficiency (PIE) curves of $1 \mathrm{MCyt}$ are measured by fixing the excitation laser at the $0_{0}^{0}+64 \mathrm{~cm}^{-1}$ band and scanning the ionization wavelength. A second frequency-doubled dye laser ( $\sim 2 \mathrm{~mJ} /$ pulse) is employed for UV/UV holeburning or depletion experiments. The holeburning (or depletion) laser pulse precedes the detection laser pulse by $300 \mathrm{~ns}$. In a $\mathrm{UV} / \mathrm{UV}$ holeburning experiment, the burning laser is fixed at the desired vibronic transition and the detection laser is scanned. The ion signal is measured at each wavelength on alternating laser shots with and without burn laser. Subtracting the unburned spectrum from the burned one yields the UV holeburning spectrum. For UV/UV depletion the detection laser is fixed at a specific vibronic transition, producing a constant ion signal. The UV depletion laser is scanned; when the depletion laser is in resonance with a vibronic transition of the same species that is being detected, the ion signal is reduced, giving a dip in the depletion spectrum.

Excited-state lifetimes and intersystem crossing (ISC) transients are measured by ns and ps excitation/delayed ionization, keeping the excitation and ionization wavelengths fixed. In the ns experiment, the time delay between the excitation and ionization laser pulses is scanned by a computer-controlled digital delay unit (1.5 ns accuracy, $60 \mathrm{ps}$ RMS jitter). The picosecond excitation/delayed ionization delay method has been described elsewhere. ${ }^{51,52}$ Briefly, the 20 ps UV excitation pulse is generated by an Ekspla PG401 OPO/optical parametric amplifier (OPA) system. The $20 \mathrm{ps}$ ionization pulses at $266 \mathrm{~nm}$ are generated by quadrupling the ps Nd:YAG pump laser, and the $213 \mathrm{~nm}$ pulses are produced by mixing the $266 \mathrm{~nm}$ with the remaining $1064 \mathrm{~nm}$. The transients and excited-state lifetimes $\tau$ are fitted with a program written in Interactive Data Language (IDL), ${ }^{53}$ see Ref. 17. The fit model includes an exponential decay for the optically excited $S_{1}$ state and an exponential increase for triplet state formed in the ISC process. The instrumental response function (IRF) is given by the convolution of the excitation and ionization laser pulses and is $7 \mathrm{~ns}$ FWHM for the ns experiment, as determined by fitting to the rise of the transient. The ps excitation/ionization IRF was measured to be 28 ps FWHM. ${ }^{15,51,52}$

\section{RESULTS AND DISCUSSION}

\section{A. Resonant two-photon ionization and UV/UV holeburning spectra}

The vibrationally resolved two-color $S_{0} \rightarrow S_{1}$ R2PI spectra of 1MCyt and 1-d $d_{3}$-MCyt in the $31250-32800 \mathrm{~cm}^{-1}$ range with ionization at $223 \mathrm{~nm}$ are shown in Figure 2. Both spectra exhibit five to six intense vibronic bands plus many weaker bands up to $\sim 500 \mathrm{~cm}^{-1}$ above the lowest-energy bands at 31852 and $31925 \mathrm{~cm}^{-1}$, respectively. The UV/UV holeburning spectra of $1 \mathrm{MCyt}$ and $1-d_{3}$-MCyt with depletion at the band at $+455 \mathrm{~cm}^{-1}$ and $+381 \mathrm{~cm}^{-1}$, respectively, reproduce the $2 \mathrm{C}$-R2PI spectra in detail, see Figures S1 and S3 (supplementary material). The holeburning spectra 


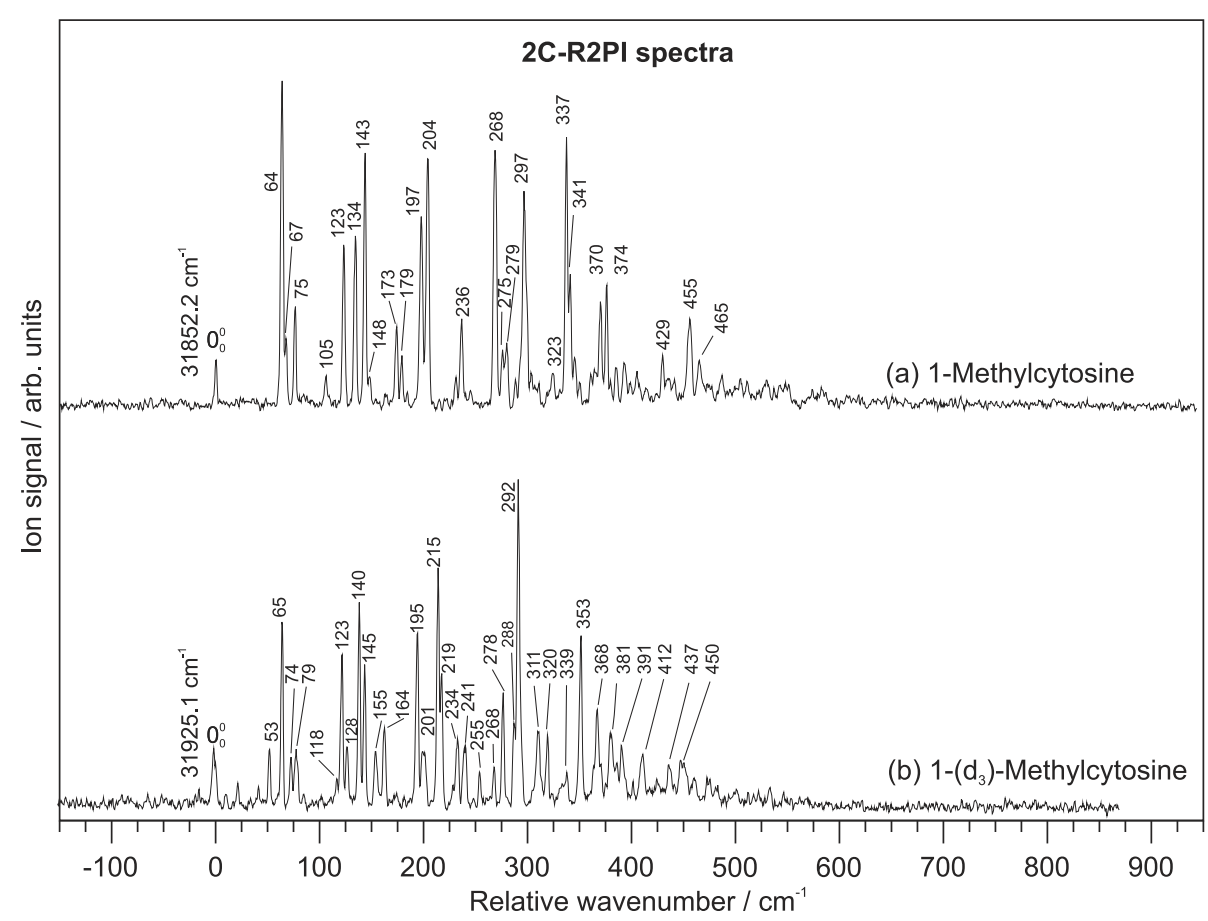

FIG. 2. Two-color resonant twophoton ionization spectra of supersonically cooled (a) 1MCyt and (b) $1-d_{3}$-MCyt (ionization at $223 \mathrm{~nm}$ ). Frequency scale relative to the $S_{0}$ $\rightarrow S_{1}$ electronic origins at $31852 \mathrm{~cm}^{-1}$ (1MCyt) and $31925 \mathrm{~cm}^{-1}$ $\left(1-d_{3}\right.$-MCyt). prove that all the vibronic bands observed in Figure 2 originate from the same tautomer and $S_{0}$ ground state level.

The electronic origins lie at $31852 \mathrm{~cm}^{-1}$ (1MCyt) and $31925 \mathrm{~cm}^{-1}\left(1-d_{3}\right.$-MCyt) with intensities that are $10 \%-20 \%$ of the strongest vibronic bands. The low Franck-Condon factors of these $0_{0}^{0}$ bands signal a marked geometry change between the $S_{0}$ and $S_{1}$ excited states. De Vries et al. assigned the intense band at $0_{0}^{0}+64 \mathrm{~cm}^{-1}$ (at $31908 \mathrm{~cm}^{-1}$ ) as the $S_{0} \rightarrow S_{1} 0_{0}^{0}$ band and attributed the origins assigned here to hot bands; they mentioned UV/UV depletion spectra but did not show them in their paper. ${ }^{9,10}$ The present UV/UV holeburning spectra call for revision of their assignment.

We also recorded the 2C-R2PI spectra of $1 \mathrm{MCyt}$ and $1-d_{3}$-MCyt with the ionization laser tuned to $215 \mathrm{~nm}$, where triplet signal is detected (see below) and with the ionization delayed by $30 \mathrm{~ns}$ relative to the excitation laser, so that only triplet molecules are ionized. The delayed R2PI spectra are identical to the prompt two-color R2PI spectra and break off at $\sim 500 \mathrm{~cm}^{-1}$ as well, suggesting that a barrier to internal conversion (IC) is exceeded at this energy level (see Figures S4 and S5 in the supplementary material).

\section{B. Vertical and adiabatic excitations}

\section{Tautomers of 1-methylcytosine}

$1 \mathrm{MCyt}$ can exist in the different tautomer/rotamer forms shown in Figure 1. The relative ground-state energies are given in Table I. All computational methods predict the amino-keto tautomer 1 as the most stable by $\geq 1.1 \mathrm{kcal} / \mathrm{mol}$, and we expect it to be the dominant species in the supersonic jet. The imino-keto-trans $\mathbf{3 a}$ and imino-keto-cis $\mathbf{3 b}$ tautomers are $1.1-3.0$ and $1.8-4.7 \mathrm{kcal} / \mathrm{mol}$ higher, respectively. The imino-enol tautomers and rotamers are even higher, by
$20 \mathrm{kcal} / \mathrm{mol}$ or more. To assess the putative contributions of the tautomers $\mathbf{3 a}$ and $\mathbf{3 b}$ to the spectra, we calculated their lowest ${ }^{1} \pi \pi^{*}$ adiabatic transition energies. At the TD-B3LYP level, these are $2050-2700 \mathrm{~cm}^{-1}$ higher than that of the aminoketo form 1. We therefore only consider tautomer $\mathbf{1}$ from now on.

\section{Excitation spectrum}

Table II shows the SCS-CC2/aug-cc-pVDZ and MSCASPT2(14,10)/ANO-L calculated adiabatic and vertical

TABLE I. Calculated ground-state energies (in $\mathrm{kcal} / \mathrm{mol}$ ) of the seven tautomers/rotamers of $1 \mathrm{MCyt}$ at various levels of theory, relative to the aminoketo 1 tautomer.

\begin{tabular}{|c|c|c|c|c|c|c|c|}
\hline Method/basis & 1 & $\mathbf{3 a}$ & $\mathbf{3 b}$ & 1.1 & 1.2 & 1.3 & 1.4 \\
\hline B3LYP/TZVP & 0.00 & 2.14 & 3.97 & 30.49 & 20.44 & 23.96 & 34.98 \\
\hline SCS-CC2/aug-cc-pVDZ & 0.00 & 1.61 & 3.28 & & & & \\
\hline SCS-MP2/aug-cc-pVTZ & 0.00 & 1.12 & 2.83 & & & & \\
\hline $\mathrm{G} 3 \mathrm{MP} 2 \mathrm{~B} 3 / \mathrm{L}^{\mathrm{a}}$ & 0.00 & 1.76 & 1.76 & & & & \\
\hline MS-CASPT2/6-31G*b & 0.00 & 2.31 & 3.92 & & & & \\
\hline $\mathrm{MP} 2 / 6-311 \mathrm{G} * * \mathrm{c}$ & 0.00 & 1.78 & 3.54 & & & & \\
\hline B3LYP/6-31+G*d & 0.00 & 2.9 & 4.6 & & 21.4 & 24.8 & \\
\hline B3-MP2/6-311++G(df,2p) $)^{e}$ & 0.00 & 2.94 & 4.73 & & & & \\
\hline B3LYP/6-31++G**f & 0.00 & 2.53 & 4.34 & & & & \\
\hline MP2/aug-cc-pVDZ & 0.00 & 2.34 & 4.09 & & & & \\
\hline $\operatorname{CCSD}(T) /$ aug-cc-pVDZ ${ }^{\text {f,g }}$ & 0.00 & 1.42 & 3.09 & & & & \\
\hline
\end{tabular}

${ }^{\mathrm{a}}$ Reference 14 .

${ }^{\mathrm{b}}$ Reference 35 (CASPT2(16,12)/ANO-S//CASSCF(14,10)/6-31G*.

${ }^{\mathrm{c}}$ Reference 56 .

${ }^{\mathrm{d}}$ Reference 57.

${ }^{\mathrm{e}}$ Reference 58 (Single-point energies with B3LYP/6-31+G(d,p) zero-point energy corrections).

${ }^{\mathrm{f}}$ Reference 59 .

${ }^{\mathrm{g}}$ Single-point energies on the MP2/aug-cc-pVDZ structure. 
TABLE II. SCS-CC2 and MS-CASPT2 calculated adiabatic and vertical transition energies (in $\mathrm{cm}^{-1}$ ) and oscillator strengths $f_{e l}$ for the amino-keto 1 tautomer of $1 \mathrm{MCyt}$.

\begin{tabular}{|c|c|c|c|c|c|}
\hline \multirow[b]{2}{*}{ State } & \multicolumn{2}{|c|}{ SCS-CC2/aug-cc-pVDZ } & \multicolumn{2}{|c|}{ MS-CASPT2/ANO-L ${ }^{b}$} & \multirow{2}{*}{$\begin{array}{c}\text { Experimental } \\
\text { Adiab. }\end{array}$} \\
\hline & Vertical $\left(f_{e l}^{\mathrm{a}}\right)$ & Adiab. & Vertical $\left(f_{e l}^{\mathrm{a}}\right)$ & Adiab. & \\
\hline$S_{1}\left({ }^{1} \pi \pi^{*}\right)$ & $37049(0.0876)$ & 31899 & $37449(0.1035)$ & $31429^{c}$ & $31852 \pm 1$ \\
\hline$S_{2}\left({ }^{1} n \pi^{*}\right)^{\mathrm{d}}$ & $42537(0.0066)$ & 36948 & $42411(0.0028)$ & & \\
\hline$S_{3}{ }^{\mathrm{e}}$ & $44169(0.0155)$ & & $45870(0.0091)$ & & \\
\hline$S_{4}{ }^{\mathrm{e}}$ & $45578(0.0599)$ & & $46098(0.0770)$ & & \\
\hline$T_{1}\left({ }^{3} \pi \pi^{*}\right)$ & 30668 & 28563 & 31560 & 27862 & $25600 \pm 500$ \\
\hline$T_{2}\left({ }^{3} \pi \pi^{*}\right)$ & 38121 & & 39167 & & \\
\hline$T_{3}\left({ }^{3} n \pi^{*}\right)$ & 41231 & & 41299 & $33529^{f}$ & \\
\hline Ion ground state $\left(D_{0}\right)$ & 77043 & 71904 & & & $68350 \pm 200$ \\
\hline
\end{tabular}

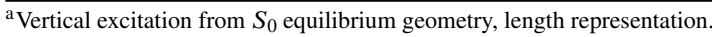

${ }^{\mathrm{b}}$ At CAM-B3LYP optimized geometries, details see text.

${ }^{\mathrm{c}}$ Includes ZPE correction of $-1013 \mathrm{~cm}^{-1}$ calculated at the CAM-B3LYP/6-311G** level of theory.

${ }^{\mathrm{d}}$ Excitation mainly from the oxygen lone pair.

${ }^{\text {e}}$ States with mixed ${ }^{1} \pi \pi * /{ }^{1} n \pi *$ character.

${ }^{\mathrm{f}}$ State becomes $T_{2}$ upon optimization, excitation mainly from the nitrogen lone pair.
}

transition energies for the amino-keto 1 tautomer. Both the SCS-CC2 and the MS-CASPT2 methods predict that the $S_{1}$ state is predominantly of ${ }^{1} \pi \pi^{*}$ character. At the SCS-CC2 level, $S_{1}$ also has $17 \% n \pi^{*}$ character. The adiabatic $S_{0} \rightarrow S_{1}$ transition energies are $31899 \mathrm{~cm}^{-1}$ and $31429 \mathrm{~cm}^{-1}$ at the SCS-CC2 and the MS-CASPT2 levels, respectively, in very good agreement with the experimentally observed $0_{0}^{0}$ band at $31852 \mathrm{~cm}^{-1}$, see Figure 2. The calculated vertical excitations are in line with previous calculations. ${ }^{14,54}$ The SCS-CC2 and MS-CASPT2 methods predict the lowest vertical ${ }^{1} \pi \pi^{*}$ transition at $37049 \mathrm{~cm}^{-1}$ and $37449 \mathrm{~cm}^{-1}$, respectively, with oscillator strengths $f_{e l} \sim 0.1$. This is in good agreement with the experimental value of $37500 \mathrm{~cm}^{-1}$ for cytosine obtained from electron energy loss spectroscopic measurements. ${ }^{55}$ The lowest ${ }^{1} n \pi^{*}$ state, $S_{2}$, appears at approximately $42500 \mathrm{~cm}^{-1}$ with both methods. Finally, $S_{3}$ and $S_{4}$ at the MS-CASPT2 level appear as two close-lying transitions around $46000 \mathrm{~cm}^{-1}$ with mixed ${ }^{1} \pi \pi^{*}$ and ${ }^{1} n \pi^{*}$ character.

\section{Excited-state structures}

The structure of the $S_{1}$ minimum, $\left(\pi \pi^{*}\right)_{\text {Min }}$, depends on the level of theory used for the optimization. A quasi-planar minimum, pyramidalized at $\mathrm{C}_{6}$, is obtained with the $\operatorname{CASSCF}(10,8) / 6-311 \mathrm{G}^{* *}$, MS-CASPT2 $(8,7) / \mathrm{DZP},{ }^{36}$ CC2/aug-cc-pVDZ, and TD-B3LYP/TZVP methods, whereas a ring-puckered structure is obtained at the $\operatorname{CASSCF}(12,11) / 6$ $311 \mathrm{G}^{* *}$, SCS-CC2/aug-cc-pVDZ, and TD-CAM-B3LYP/6$311 \mathrm{G}^{* *}$ levels. The different $S_{1}$ minima have also different bond lengths. For instance, the $\mathrm{C}_{2}-\mathrm{O}_{7}$ bond length varies from $1.255 \AA$ with TD-CAM-B3LYP to $1.320 \AA$ with CASSCF $(12,11)$ and $1.354 \AA$ with SCS-CC2 (see the supplementary material for details). Good agreement with the experimental adiabatic transition of $31852 \mathrm{~cm}^{-1}$ is obtained at the TD-B3LYP, MS-CASPT2 $(8,7)$, and SCS-CC2 levels of theory. However, the three methods give substantially different structures, and the main question is whether the $0_{0}^{0}$ transition leads to a quasi-planar structure, as suggested by B3LYP and MS-CASPT2 $(8,7)$, or a ring-puckered structure as suggested by SCS-CC2 and TD-CAM-B3LYP. To solve this question, the energies of all structures were recalculated at the MS-CASPT2(14,10)/ANO-L level. The lowest $S_{1}$ energy at this level is obtained for the TD-CAM-B3LYP and $\operatorname{CASSCF}(12,11)$ optimized structures, for which the ring is puckered. The relative potential energies without ZPE correction are 32442 and $32771 \mathrm{~cm}^{-1}$, respectively. Thus, the SCS-CC2 and the MS-CASPT2 $(14,10)$ calculations on the structures optimized with different methods agree that $\left(\pi \pi^{*}\right)_{\text {Min }}$ has a ring-puckered structure. This agrees with the small intensity of the experimental $0_{0}^{0}$ transition, see above, and with the large Franck-Condon factors in out-of-plane vibrations, see below. The overlay of the $S_{0}$ and $S_{1}$ minima obtained at the SCS-CC2 level (Figure 3) shows that, together with the ring puckering, the methyl group undergoes a rotation upon $S_{0}$ $\rightarrow S_{1}$ excitation of $48^{\circ}$ (SCS-CC2) and $60^{\circ}$ (TD-B3LYP), see also Section III F.

The large variations of the minimum structures predicted by the different theoretical methods point to the importance of vibronic (or pseudo-Jahn-Teller) interactions between the close-lying ${ }^{1} \pi \pi^{*}$ and ${ }^{1} n \pi^{*}$ states, which leads to breaking of the ring planarity and puckering of the ring. ${ }^{60,61}$ The ${ }^{1} \pi \pi^{*} \leftrightarrow{ }^{1} n \pi^{*}$ vibronic interaction is the cause for the differences found between similar $a b$ initio methods. Thus, SCS-CC2 and $\operatorname{CASSCF}(12,11)$ predict ring-puckered structures, whereas unscaled CC2 and CASSCF with a smaller $(8,7)$ active space give a quasi-planar structure. In the coupled cluster case, SCS-CC2 is more suitable for cases with high electronic correlation such as the present one. In the CASSCF case, the $(12,11)$ active space was specially tailored to account for the vibronic interaction since it includes the oxygen lone pair responsible for the ${ }^{1} n \pi^{*}$ excitation and a $3 p$ orbital on the oxygen to account for radial correlation. For the same reason, the MS-CASPT2 optimization with the reduced $(8,7)$ active space ${ }^{36}$ which lacks the oxygen lone pair responsible for the interaction gives a quasi-planar structure whose MS-CASPT2 $(14,10)$ energy is approximately $800 \mathrm{~cm}^{-1}$ higher than the TD-CAM-B3LYP optimized structure. 

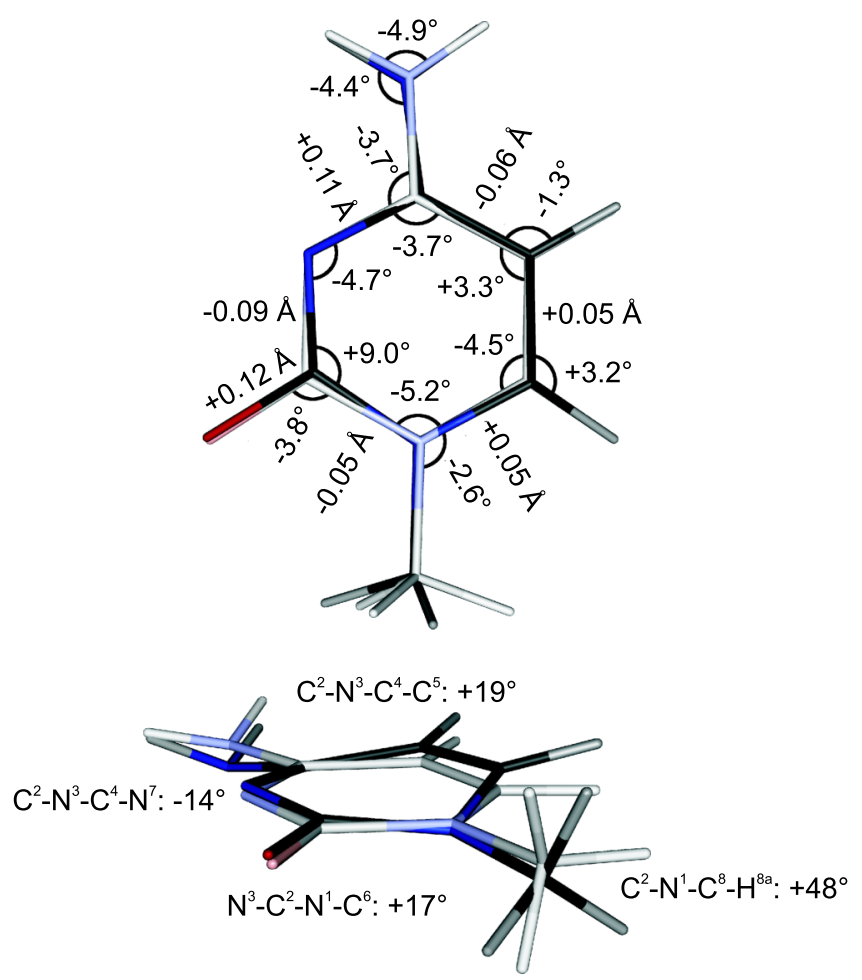

FIG. 3. SCS-CC2/aug-cc-pVDZ calculated geometry changes of $1 \mathrm{MCyt}$ upon ${ }^{1} \pi \pi^{*}$ excitation; the excited state is in darker color. Only bond length changes $\geq 0.05 \AA$ and angle changes $\geq 1^{\circ}$ are indicated.

\section{Nanosecond excitation/delayed ionization measurements}

Figure 4(a) shows the nanosecond excitation/delayed ionization transients for $1 \mathrm{MCyt}$ and $1-d_{3}-\mathrm{MCyt}$, measured at the $0_{0}^{0}+64 \mathrm{~cm}^{-1}$ and $0_{0}^{0}+65 \mathrm{~cm}^{-1}$ bands with ionization at $223 \mathrm{~nm}$. The initial peak ("prompt" ionization signal) corresponds to ionization of the optically excited $S_{1}\left({ }^{1} \pi \pi^{*}\right)$ state. The nearly flat "tail" extending to longer delay time corresponds to the ionization of a long-lived state, which is shown to be the $T_{1}$ triplet state in Section III D. The ns excitation/ionization transient for $1-d_{3}$-MCyt is similar to 1-MCyt, the long-lived component being $11 \%$ higher.

Figure 4(b) shows the dependence of the ns transient for 1MCyt on the ionization wavelength. For ionization wavelengths $>240 \mathrm{~nm}$ only prompt ionization signal is observed; with decreasing ionization wavelength (increasing ionization energy) the delayed signal increases relative to the prompt signal, reaching 50\% of the prompt signal at $215 \mathrm{~nm}$.

The percentages of triplet ion signal are noted above the delayed transient in Figure 4(b); they are related to the ionization cross section $\sigma_{i o n}^{+}$of the triplet state relative to the $S_{1}$ state, as will be discussed in Section III D. In Section III E we show that the $S_{1}$ state lifetime at $0_{0}^{0}+64 \mathrm{~cm}^{-1}$ is $\sim 10$ times shorter than the $5 \mathrm{~ns}$ pulse lengths of the excitation and ionization lasers. In contrast, the $T_{1}$ lifetime is $\sim 200 \mathrm{~ns}$ and thus much longer than the laser pulse length. Hence, the efficiency with which a given $S_{1}$ vibronic level is ionized depends on its lifetime, relative to the $5 \mathrm{~ns}$ laser pulse length, so the detection efficiencies decrease for the higher $S_{1}$

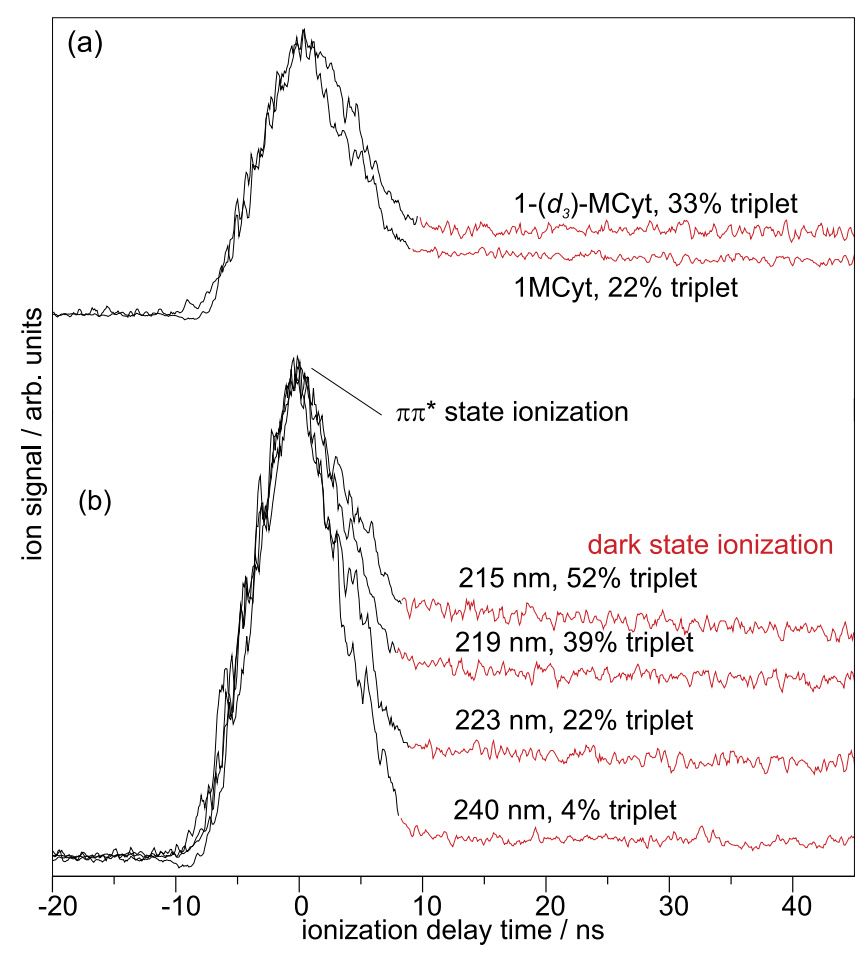

FIG. 4. (a) Ionization delay curves for $1 \mathrm{MCyt}$ and $1-d_{3}$-MCyt excited at the $0_{0}^{0}+64 \mathrm{~cm}^{-1}$ band $\left(31916 \mathrm{~cm}^{-1}\right)$ and $0_{0}^{0}+65 \mathrm{~cm}^{-1}$ band $\left(31992 \mathrm{~cm}^{-1}\right)$, with ionization at $223 \mathrm{~nm}$. (b) Nanosecond ionization delay curves of 1-MCyt for the series of ionization wavelengths $215-240 \mathrm{~nm}$.

vibronic levels with shorter lifetimes (see Section III E). The relative singlet and triplet ion signals in the ns pump/ionization transients depend on the lifetime of the $S_{1}$ level and on the ratio of the ionization cross sections $\sigma_{\text {ion }}^{+}\left(S_{1}\right) / \sigma_{\text {ion }}^{+}\left(T_{1}\right)$, both must be taken into account when modeling the ns excitation/ionization transients, as previously discussed for cytosine.${ }^{17}$ We discuss the fitting of the ISC rates in combination with the ps excitation/ionization transients in Section III E.

\section{Photoionization efficiency curves and thresholds}

Figure 5 shows two photoionization efficiency (PIE) curves of 1-methylcytosine as a function of ionization laser energy, both measured when exciting at the $0_{0}^{0}+64 \mathrm{~cm}^{-1}$ band $\left(31852 \mathrm{~cm}^{-1}\right)$. The black curve is measured at the prompt ionization peak, corresponding to ionization of the $S_{1}$ state. The red curve is measured with the ionization pulse delayed by $50 \mathrm{~ns}$, corresponding to ionization of the longlived state. The relative heights of the prompt and delayed PIE curves are scaled according to the ratio of the prompt and $50 \mathrm{~ns}$ delayed signals in Figure 4. The $S_{1}$ state ionization threshold is observed at $36000 \pm 200 \mathrm{~cm}^{-1}$; the ion signal rises slowly above the background, does not exhibit a steplike onset, and thus is an upper limit to the adiabatic ionization energy. The sum of the excitation and the ionization threshold corresponds to a ionization energy $I E=67850 \pm 200 \mathrm{~cm}^{-1}$ or $8.41 \pm 0.03 \mathrm{eV}$. Compared to amino-keto cytosine which has an adiabatic ionization energy IE of $8.73 \pm 0.02 \mathrm{eV},{ }^{16}$ the methyl substitution at $\mathrm{N}_{1}$ lowers the ionization energy by at least $0.32 \mathrm{eV}$. 


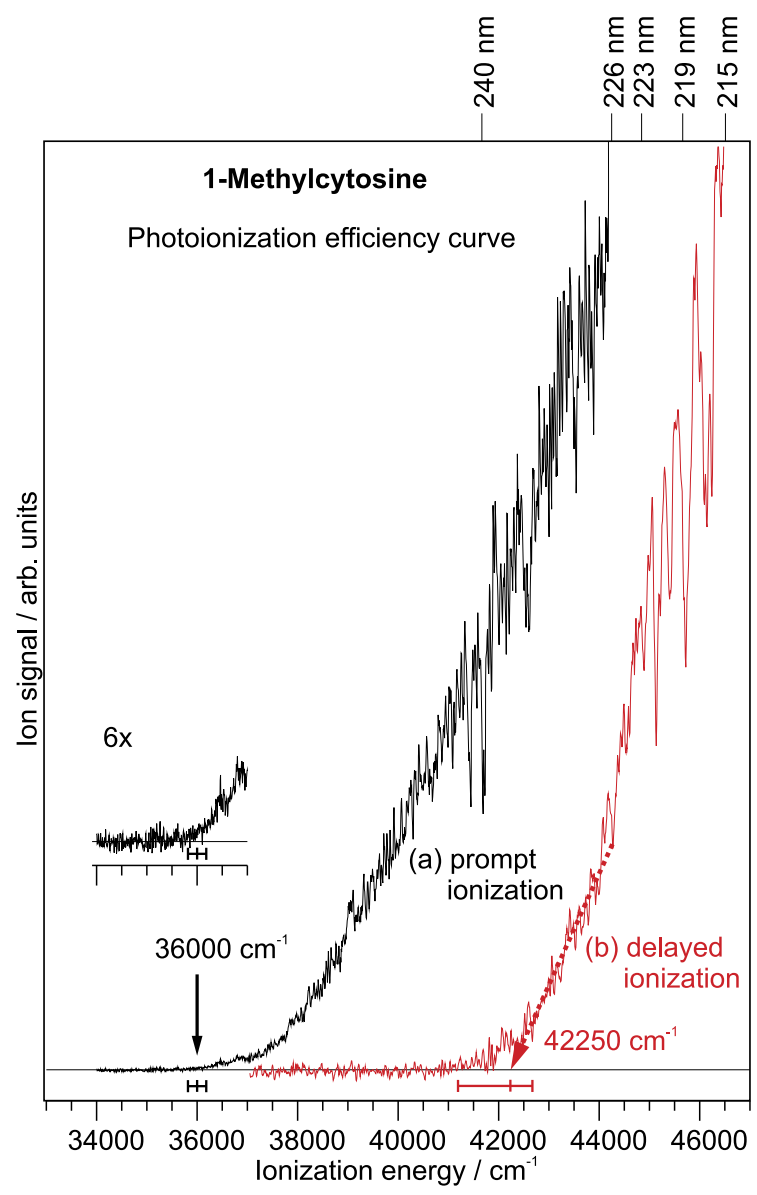

FIG. 5. Photoionization efficiency curves for (a) prompt ( $0 \mathrm{~ns}$ delay) and (b) $50 \mathrm{~ns}$ delayed ionization of $1 \mathrm{MCyt}$, when exciting the $0_{0}^{0}+64 \mathrm{~cm}^{-1}$ band. Uncertainties indicated with horizontal bars.

The onset of the delayed-ionization PIE curve in Figure 5 at $\sim 41200 \mathrm{~cm}^{-1}$ is $5200 \mathrm{~cm}^{-1}$ above the $S_{1}$ threshold, with a slower initial rise above the background. If we assume that the $5200 \mathrm{~cm}^{-1}$ correspond to the adiabatic energy difference between $S_{1}$ and the lower-lying long-lived state, this energy difference is randomized over the 42 vibrational modes of $1 \mathrm{MCyt}$ after the $50 \mathrm{~ns}$ delay time. We thus interpret the sluggish onset of the delayed-ionization PIE curve between $41000-42250 \mathrm{~cm}^{-1}$ as photoionization from vibrationally hot triplet states that contribute ion signal below the "cold" ionization threshold. Similar to the procedure in Ref. 16, we estimate the cold ionization threshold by the backextrapolation of the linear rise of the PIE curve to the zeroion-signal background, giving $42250 \pm 500 \mathrm{~cm}^{-1}$. Comparing this estimate of the ionization energy of the long-lived state to the IE measured via the $S_{1}$ state implies that the long-lived state lies about $25600 \pm 500 \mathrm{~cm}^{-1}(3.17 \pm 0.06 \mathrm{eV})$ above the $S_{0}$ state.

Table II shows that of all the calculated excited states of 1MCyt, only $T_{1}\left({ }^{3} \pi \pi^{*}\right)$ has approximately this energy, hence we consider the assignment of the long-lived dark state as established. Also, the $T_{1}$ state should have a long lifetime, as is observed experimentally. The SCS-CC2 $\left(28563 \mathrm{~cm}^{-1}\right)$ and the MS-CASPT2 $\left(27862 \mathrm{~cm}^{-1}\right) T_{1}\left({ }^{3} \pi \pi^{*}\right)$ energies are about 3000 and $2200 \mathrm{~cm}^{-1}$ higher, respectively, than the experimental value. At the MS-CASPT2 level of theory, the adiabatic energy of $T_{2}$ which has $\left(n \pi^{*}\right)$ character is $33529 \mathrm{~cm}^{-1}$ (see Table II).

Comparison of the $I E=67850 \pm 200 \mathrm{~cm}^{-1}$ with the vertical and adiabatic calculated ionization energies in Table III shows that (1) the SCS-CC2 ionization energies are consistently higher than those calculated with all other methods, (2) the experimental IE is about $1000 \mathrm{~cm}^{-1}$ higher than the B3LYP calculated ionization energies, and (3) about $1000 \mathrm{~cm}^{-1}$ lower than the vertical IEs from photoelectron spectra, P3 electron propagator/6-311G**, and OVGF-MP2/6$311 \mathrm{G}(\mathrm{d}, \mathrm{p})$ calculations. ${ }^{56,62,63}$ Since a large geometry change is expected for the $S_{1} \rightarrow D_{0}$ transition, the Franck-Condon factor of the adiabatic transition in ionization is expected to be very small.

\section{E. Picosecond excitation/delayed ionization measurements}

Figure 6(a) shows the ps two-color resonant two-photon ionization overview spectrum of $1 \mathrm{MCyt}$ with ionization at $213 \mathrm{~nm}$. Note the different band intensities and overall shape, compared to the ns laser R2PI spectrum in Figure 2. While the $0_{0}^{0}$ band at $31852 \mathrm{~cm}^{-1}$ is clearly observed in the ns spectrum, it is hardly detectable in the ps R2PI spectrum. The following three bands in the ns R2PI spectrum are among the most

TABLE III. Calculated and experimental ionization energies of $1 \mathrm{MCyt}\left(\mathrm{in} \mathrm{cm}^{-1}\right)$.

\begin{tabular}{|c|c|c|c|c|c|c|}
\hline \multirow[b]{2}{*}{ Method } & \multicolumn{3}{|c|}{ Vertical } & \multicolumn{3}{|c|}{ Adiabatic } \\
\hline & $S_{0} \rightarrow D_{0}$ & $S_{1} \rightarrow D_{0}$ & $T_{1} \rightarrow D_{0}$ & $S_{0} \rightarrow D_{0}$ & $S_{1} \rightarrow D_{0}$ & $T_{1} \rightarrow D_{0}$ \\
\hline B3LYP/TZVP & 67321 & 39172 & $46217\left(T_{1}\right)$ & 66338 & 34312 & $40772\left(T_{1}\right)$ \\
\hline SCS-CC2/aug-cc-pVDZ & 77043 & 44839 & $56248\left(T_{1}\right)$ & 71904 & 40005 & $43341\left(T_{1}\right)$ \\
\hline B3LYP/6-31+G(d,p) $)^{\mathrm{a}}$ & 67920 & & & 66640 & & \\
\hline Photoelectr. spectr.(exp. $)^{d}$ & 69760 & & & & & \\
\hline
\end{tabular}

${ }^{\mathrm{a}}$ Reference 14.

${ }^{\mathrm{b}}$ Reference 56 .

${ }^{\mathrm{c}}$ Reference 62 .

${ }^{\mathrm{d}}$ Reference 63 . 


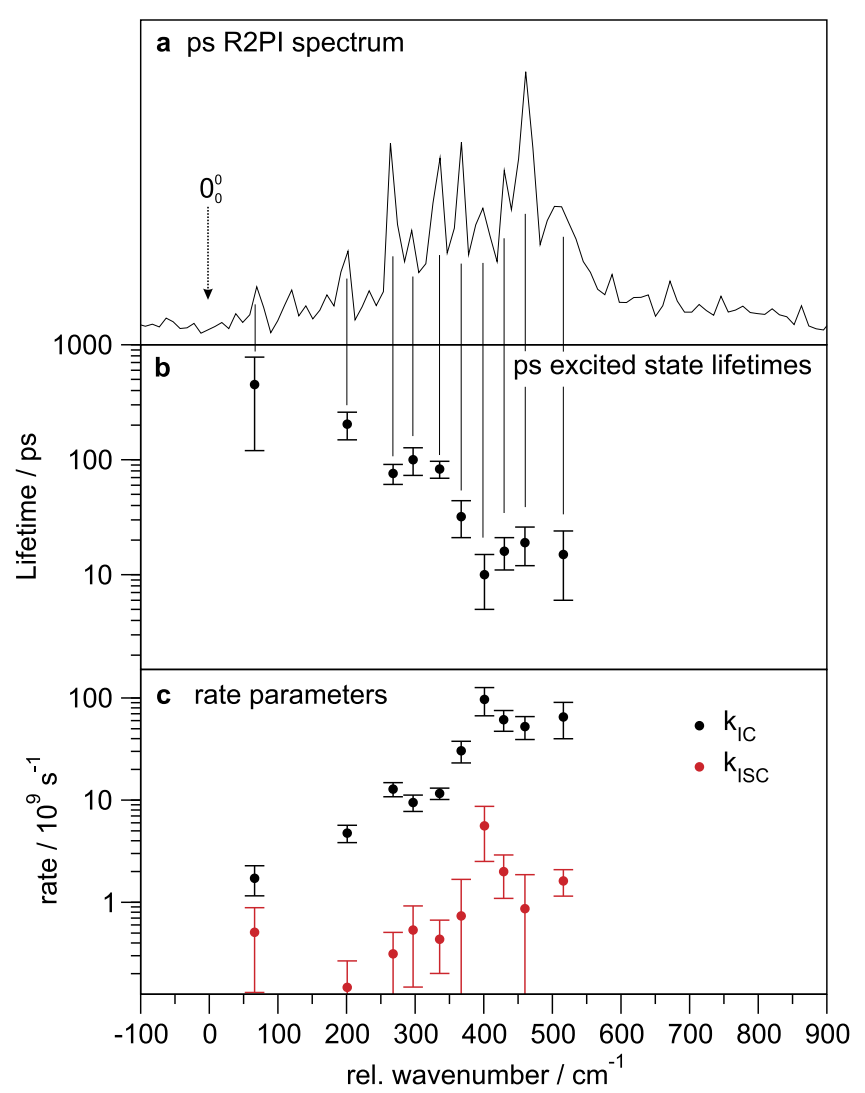

FIG. 6. (a) Picosecond R2PI spectrum of 1MCyt; (b) excited-state lifetime $\tau$, measured at the vibronic bands indicated. (c) Experimental internal conversion and intersystem crossing rate constants, see also Table IV.

intense transitions but are rather small in the ps spectrum. The band at $+460 \mathrm{~cm}^{-1}$ just before the spectrum breaks off is weak in the ns spectrum but is the most intense band in the ps R2PI spectrum. We show below that the reason for the large difference between the ns and ps R2PI spectra results from the fact that the $S_{1}$ state lifetimes decrease by a factor of $50-70$ over the vibrational excess energy range $E_{\text {exc }}=0-500 \mathrm{~cm}^{-1}$ in the $S_{1}$ state.

The $S_{1}\left({ }^{1} \pi \pi^{*}\right)$ state kinetics is modeled as

$$
\frac{d\left[S_{1}\right]}{d t}=-\left(k_{\text {rad }}^{S_{1}}+k_{I C}^{S_{1}}+k_{I S C}^{S_{1}}\right) \cdot\left[S_{1}\right],
$$

where $k_{\text {rad }}$ is the $S_{1} \rightarrow S_{0}$ radiative decay rate. The SCSCC2 calculated oscillator strength of $1 \mathrm{MCyt}$ is $f_{e l}=0.0876$, giving $\tau_{\text {rad }} \approx 12$ ns or $k_{\text {rad }}=8.3 \cdot 10^{7} \mathrm{~s}^{-1}$. The $S_{1}$ state decays nonradiatively to $S_{0}$ by internal conversion (IC) with the rate constant $k_{I C}^{S_{1}}$. Since the ${ }^{1} n \pi^{*}$ state is calculated to lie above the ${ }^{1} \pi \pi^{*}$ state, see Section III B, relaxation to the ${ }^{1} n \pi^{*}$ state is not included in the kinetic model. The third term in Eq. (1) expresses the formation of the $T_{1}$ from $S_{1}$ with the intersystem crossing (ISC) rate constant $k_{I S C}^{S_{1}}$, see Sections III C and III D. We assume that $T_{1}$ relaxes to $S_{0}$ by $T_{1} \rightsquigarrow S_{0}$ ISC as well as by phosphorescence, these two pathways are combined into a single rate constant $k_{T}$,

$$
\frac{d\left[T_{1}\right]}{d t}=k_{I S C}^{S_{1}} \cdot\left[S_{1}\right]-k_{T} \cdot\left[T_{1}\right] .
$$

The ns excitation/ionization transients show that the $T_{1}$ decay is on the $\sim 200 \mathrm{~ns}$ time scale, so $k_{T}$ is small compared
N1-Methylcytosine Excited State Lifetimes

total fit $-{ }^{1} \pi \pi^{*}-\mathrm{T}_{1}$

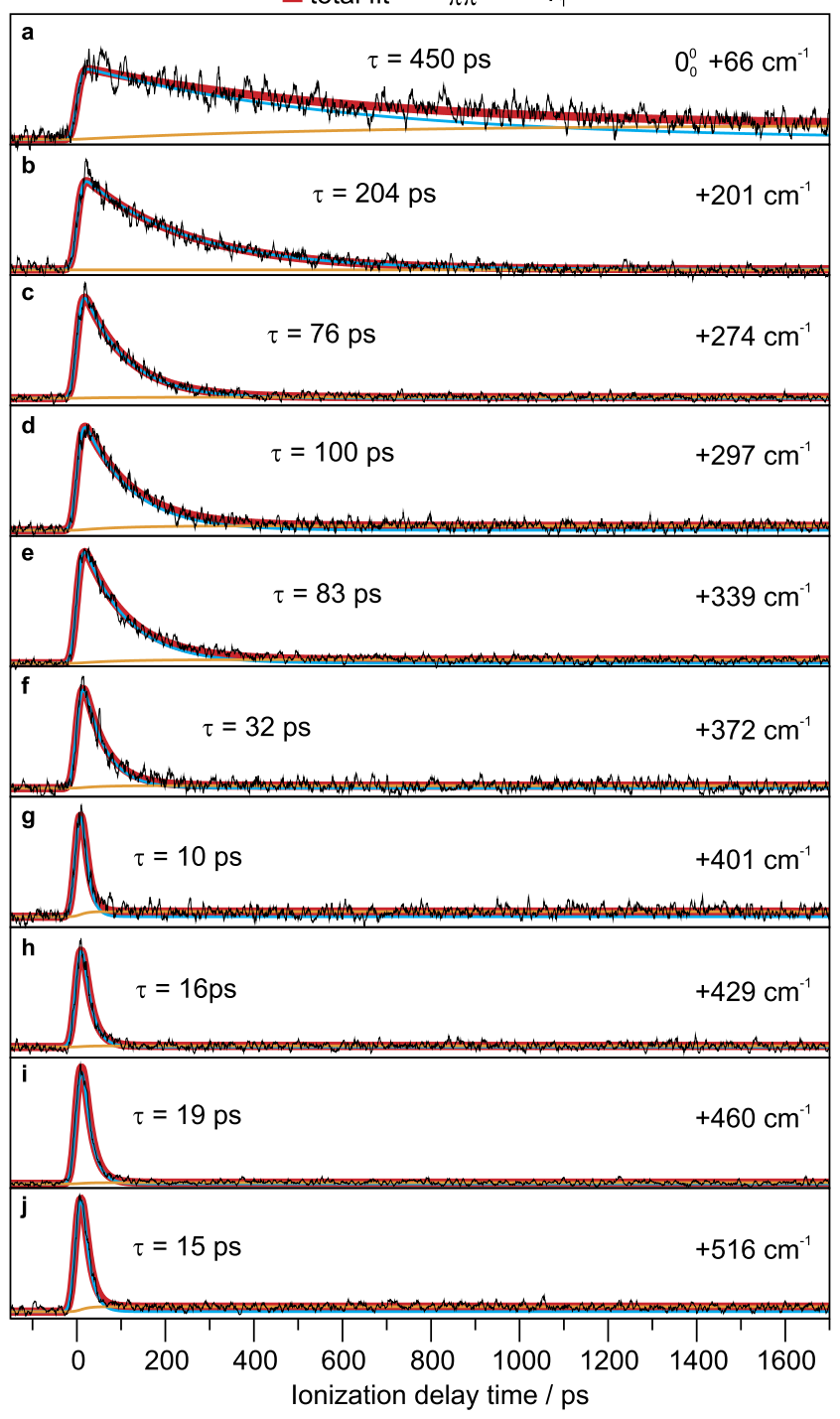

FIG. 7. Picosecond excitation/delayed ionization transients of $1 \mathrm{MCyt}$, with ionization at $213 \mathrm{~nm}$. Black: experimental, blue: $S_{1}$ decay, yellow: $T_{1}$ increase, red: total fit. The indicated wavenumbers (relative to the $0_{0}^{0}$ band) are center values of the output of the UV-OPO/OPA. Because of the $\sim 4 \mathrm{~cm}^{-1}$ step-size of the UV-OPO, these do always coincide exactly with the band maxima in the ns laser R2PI spectrum in Figure 2(a).

to the other rates and is set to zero for the fits. Using the calculated $k_{\text {rad }}$, the rate constants $k_{I S C}^{S_{1}}$ and $k_{I C}^{S_{1}}$ are least-squares fitted to the ten experimental ps pump/ionization transients that are shown in Figure 7. The fitted signal contribution from $S_{1}\left({ }^{1} \pi \pi^{*}\right)$ is plotted in blue, that from $T_{1}$ in yellow, and the sum of both populations in red. As both $k_{I S C}^{S_{1}}$ and $k_{I C}^{S_{1}}$ are longer than the inverse of the 28 ps time resolution of the ps pump-ionization experiment (Section II B), the ps transients do not need to be corrected for the relative lifetime effects.

Figure 6(b) shows the excited-state lifetimes $\tau=1 /$ $\left(k_{r a d}+k_{I C}^{S_{1}}+k_{I S C}^{S_{1}}\right)$ determined from the ps pump/ionization transient measurements at ten vibronic bands, as indicated by the vertical lines. The experimental transients are plotted in black in Figure 7. The lifetimes and $1 \sigma$ error bars in Figure 6(c) are determined by fitting four to eight transients 
TABLE IV. Intersystem crossing (ISC) and internal conversion (IC) rate constants, from fits to the ps excitation/ionization transients in Figure 7.

\begin{tabular}{lccc}
\hline \hline Band $/ \mathrm{cm}^{-1 \mathrm{a}}$ & $k_{I S C}^{S_{1}} / 10^{9} \mathrm{~s}^{-1 \mathrm{~b}}$ & $k_{I C}^{S_{1}} / 10^{9} \mathrm{~s}^{-1 \mathrm{~b}}$ & Lifetime $\tau / \mathrm{ps}^{\mathrm{b}}$ \\
\hline $0_{0}^{0}+66$ & $0.5 \pm 0.4$ & $1.7 \pm 0.6$ & $450 \pm 160$ \\
+201 & $0.2 \pm 0.1$ & $4.8 \pm 0.9$ & $204 \pm 55$ \\
+274 & $0.3 \pm 0.2$ & $12.8 \pm 2.0$ & $76 \pm 15$ \\
+297 & $0.5 \pm 0.4$ & $9.5 \pm 1.7$ & $100 \pm 27$ \\
+339 & $0.4 \pm 0.2$ & $12 \pm 2.0$ & $83 \pm 14$ \\
+372 & $0.7 \pm 0.9$ & $30 \pm 7.0$ & $32 \pm 12$ \\
+401 & $5.6 \pm 3.1$ & $100 \pm 30$ & $10 \pm 5$ \\
+429 & $2.0 \pm 0.9$ & $61 \pm 14$ & $16 \pm 5$ \\
+460 & $0.9 \pm 1.0$ & $52 \pm 13$ & $19 \pm 7$ \\
+516 & $1.6 \pm 0.5$ & $65 \pm 25$ & $15 \pm 9$ \\
\hline
\end{tabular}

${ }^{a}$ Laser excitation frequency relative to the $0_{0}^{0}$ band at $31852 \mathrm{~cm}^{-1}$.

${ }^{\mathrm{b}}$ Measurement $1 \sigma$ uncertainty; standard deviation from fitting four to eight separate transients.

at the same wavelength. The $S_{1} 0_{0}^{0}$ lifetime could not be measured due to the weakness of this band, see Figure 6(a). The lowest-energy vibronic band in the ps R2PI spectrum at $0_{0}^{0}+66 \mathrm{~cm}^{-1}$ is also weak. We have recently reported the lifetime as $\tau=600 \pm 290 \mathrm{ps},{ }^{15}$ but by measuring 8 separate transients on different days we have decreased the uncertainty by nearly a factor of two, the revised value is $\tau=450 \pm 160 \mathrm{ps}$. At $+200 \mathrm{~cm}^{-1}$, the lifetime decreases to $230 \mathrm{ps}$ and continues to decrease up to the shortest measurable lifetime $\tau=15 \mathrm{ps}$ at $400 \mathrm{~cm}^{-1}$ above the $0_{0}^{0}$ band. Table IV summarizes the fitted internal conversion and intersystem crossing rate constants, as well as the corresponding lifetimes $\tau=1 /\left(k_{\text {rad }}+k_{I C}^{S_{1}}+k_{I S C}^{S_{1}}\right)$.

Contrary to the ns transients shown in Figure 4, the ps transients exhibit only a small signal increase from the growing-in of the $T_{1}$ state population. Figure 6(c) and Table IV show that in the nonradiative relaxation of 1MCyt, ISC is only significant for the $0_{0}^{0}+64 \mathrm{~cm}^{-1}$ level, at which $k_{I C}^{S_{1}}$ is relatively small and $k_{I S C}$ is only three times lower than $k_{I C}$. For the following vibronic levels, the rise in $k_{I C}$ by up to $30-40 \times$ overwhelms the slower rise in $k_{I S C}$. The knowledge of the single vibronic-level lifetimes now allows to explain the marked difference between the ps and ns R2PI spectra. Figure 8(a) shows the ns R2PI spectrum, see also Fig. 2(a). Figure 8(b) plots an exponential model function that was nonlinear least-squares fitted to the vibronic band lifetimes of Figure 6 and Table IV. Since the probability of ionizing the $S_{1}$ state vibronic levels with a 5 ns ionization pulse decreases with the decreasing lifetime of the state, we can remove the ionization bias of the ns R2PI spectrum by dividing it by the red lifetime curve in Figure 8(b). The resulting lifetime-corrected ns R2PI spectrum is shown in Figure 8(c) and compared to the experimental ps R2PI spectrum in Figure 8(d). Apart from the $\sim 20$ times lower resolution of the ps spectrum, one observes a good agreement of the two spectra up to about $+550 \mathrm{~cm}^{-1}$. The two advantages of the lifetime-corrected ns R2PI spectrum are that (1) this procedure gives correct vibronic band intensities (Franck-Condon factors) which can be compared to those derived from ab initio calculations and (2) the spectral information is obtained at the $\sim 0.5 \mathrm{~cm}^{-1}$ resolution of the ns laser and not at the much lower resolution of the ps laser.

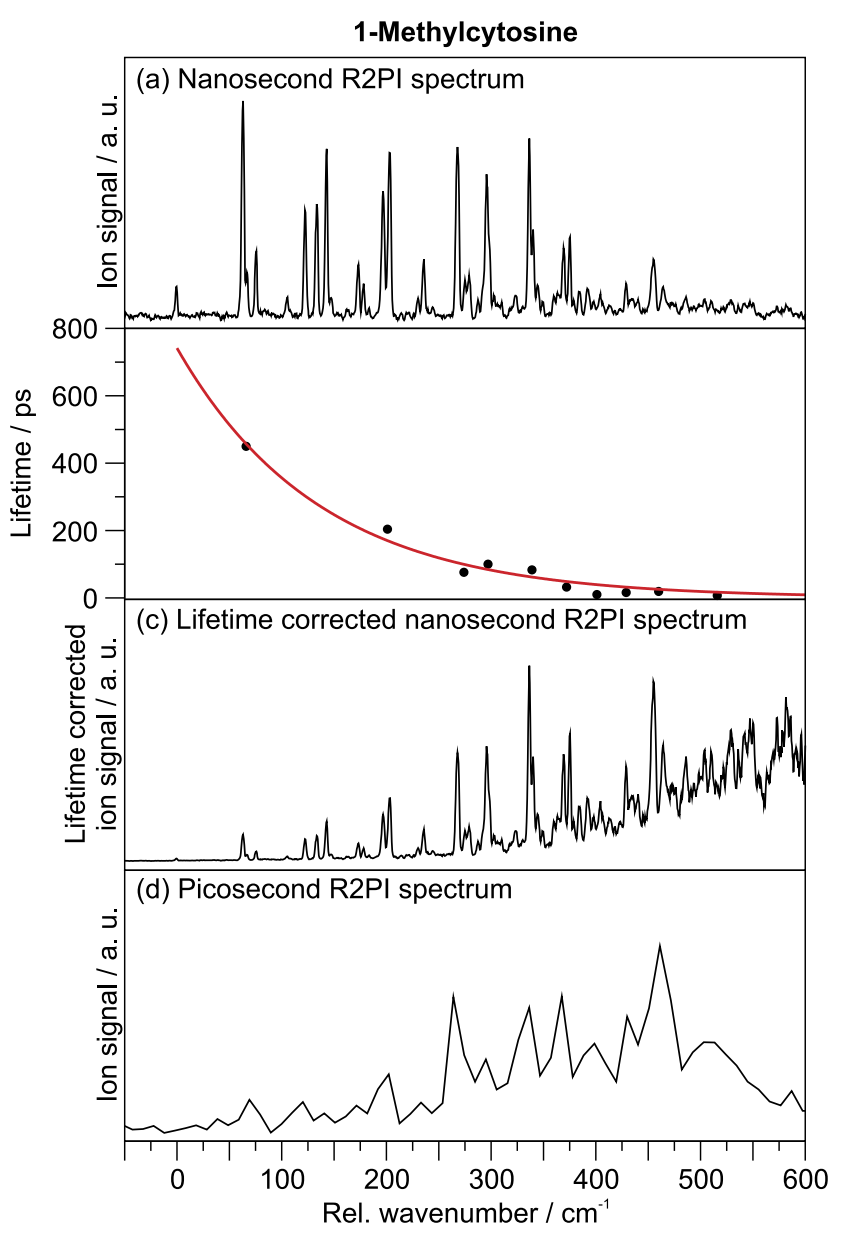

FIG. 8. (a) Nanosecond R2PI spectrum of 1MCyt, ionization at $226 \mathrm{~nm}$. (b) Excited-state lifetimes, red line is an interpolation of lifetimes $\tau$ at the corresponding bands in Figure 6(b). (c) Lifetime-corrected nanosecond R2PI spectrum. (d) Picosecond R2PI spectrum of 1MCyt, see Figure 6(a).

\section{F. Methyl group internal rotation}

As pointed out in Section III B, the $S_{1}$ minimum of $1 \mathrm{MCyt},\left(\pi \pi^{*}\right)_{\text {Min }}$, shows strong ring-puckering and the methyl group is rotated by $48^{\circ}-60^{\circ}$ relative to the $S_{0}$ minimum at the CASSCF(12,11)/6-311G**, TD-CAM-B3LYP/6-311G**, and SCS-CC2/aug-cc-pVDZ levels of theory. This suggests large displacements along at least one out-of-plane vibration and the methyl torsional mode. To assign the spectra of $1 \mathrm{MCyt}$ and $1-d_{3}$-MCyt, we performed a PGOPHER vibronic simulation, see Sec. III G. However, the methyl-rotor transitions are highly anharmonic and were treated using a one-dimensional (1D) internal-rotation Hamiltonian in the $S_{0}$ and $S_{1}\left({ }^{1} \pi \pi^{*}\right)$ states.

We define the methyl torsional angle $\phi$ as the dihedral angle $\mathrm{H}_{a}-\mathrm{C}_{2}-\mathrm{N}_{1}-\mathrm{C}_{8}$, see Figure 1. Starting from the respective minimum-energy structures, the $1 \mathrm{D}$ torsional potentials were calculated by incrementing $\phi$ in steps of $10^{\circ}$, optimizing all other structure parameters at each step. In the SCS-CC2 $S_{1}$ state, the $\mathrm{N}^{3}-\mathrm{C}^{2}-\mathrm{N}^{1}-\mathrm{C}^{6}$ and $\mathrm{C}^{6}-\mathrm{C}^{5}-\mathrm{C}^{4}-\mathrm{N}^{3}$ ring dihedrals had to be fixed to obtain a potential describing a pure torsional motion. This suggests a coupling of the methyl rotation to one or more out-of-plane modes, see below. The methyl rotor potentials are well described by the function 
TABLE V. Calculated methyl rotor parameters of 1MCyt and 1- $d_{3}$-MCyt. All values in $\mathrm{cm}^{-1}$ except $\varphi$.

\begin{tabular}{lccccc}
\hline \hline \multirow{2}{*}{ Parameter } & B3LYP & & SCS-CC2 & & MS-CASPT2 $(10,8)^{\mathrm{a}}$ \\
\cline { 2 - 3 } \cline { 5 - 6 } \cline { 5 - 6 } & TZVP & & aug-cc-pVDZ & & ANO-L \\
\hline$F_{\text {red }}^{\prime \prime}\left(h_{3}\right) / F_{\text {red }}^{\prime \prime}\left(d_{3}\right)$ & $5.42 / 2.77$ & & $5.29 / 2.71$ & & \\
$F_{\text {red }}^{\prime}\left(h_{3}\right) / F_{\text {red }}^{\prime}\left(d_{3}\right)$ & $5.40 / 2.77$ & & $5.28 / 2.70$ & & \\
$V_{3}^{\prime \prime}$ & 188 & & 361 & & 340 \\
$V_{3}^{\prime}$ & 606 & & $591 / 630^{\mathrm{b}}$ & & 500 \\
$\varphi(\operatorname{deg})$ & 60 & & $48 /-49^{\mathrm{b}}$ & & 61 \\
\hline \hline
\end{tabular}

${ }^{a}$ Single point energies on $\operatorname{CASSCF}(10,8) / 6-311 \mathrm{G}^{* *}$ optimized geometries.

${ }^{\mathrm{b}}$ Values for the $u p$-down and up-up ring-puckering conformers, respectively. See Figures 9(a) and 9(b). To obtain a potential of a pure methyl torsional motion, the $\mathrm{N}^{3}-\mathrm{C}^{2}-\mathrm{N}^{1}-\mathrm{C}^{6}$ and $\mathrm{C}^{6}-\mathrm{C}^{5}-\mathrm{C}^{4}-\mathrm{N}^{3}$ dihedrals (butterfly angle) had to be fixed, all other parameters were optimized.

$V(\phi)=V_{3} \cdot(1-\cos (3 \phi+\varphi)) / 2$, where $\varphi$ is the phase shift of the $S_{1}$ relative to the $S_{0}$ potential. $V^{\prime \prime}(\phi)$ and $V^{\prime}(\phi)$ were fitted to the calculated $S_{0}$ and $S_{1}$ energies; they are shown in Figure S6 in the supplementary material. The reduced internal-rotation constants $F_{\text {red }}$ were calculated according to the literature. ${ }^{64}$ The torsional barriers $V_{3}$, reduced internal-rotation constants $F_{\text {red }}$, and phase shifts $\varphi$ are summarized in Table $\mathrm{V}$ at various levels of theory.

The torsional eigenfunctions, eigenvalues, and FranckCondon factors were obtained by numerically solving the $1 \mathrm{D}$ torsional Hamiltonian with a home-written IDL $^{53}$ program based on the discrete variable representation, using the libraries of Dimeo. ${ }^{65}$ To calculate the contribution of the methyl rotor bands to the spectrum, a ground state barrier of $200 \mathrm{~cm}^{-1}$ and the SCS-CC2 calculated internal-rotation constants $F_{r e d}^{\prime \prime}$ and $F_{r e d}^{\prime}$ for 1MCyt and 1- $d_{3}$-MCyt, respectively, were used as an input and the excited state barriers were varied between 80 and $610 \mathrm{~cm}^{-1}$. However, we could not find a barrier height that allows a satisfactory assignment of the methyl internal-rotor bands for both molecules. This suggests that the methyl torsion cannot be treated one-dimensionally, but that the methyl rotation is coupled to other out-of-plane modes, as predicted by the SCS-CC2 method. This provides indirect support for our assignment of the non-planar structure of $\left(\pi \pi^{*}\right)_{\text {Min }}$.

To understand the methyl rotor/out-of-plane coupling, a two-dimensional (2D) $S_{1}$ state potential was calculated at the SCS-CC2/aug-cc-pVDZ level. We define the butterfly coordinate as the two dihedrals $\mathrm{N}_{3}-\mathrm{C}_{2}-\mathrm{N}_{1}-\mathrm{C}_{6}$ and $\mathrm{C}_{6}-\mathrm{C}_{5}-\mathrm{C}_{4}-\mathrm{N}_{3}$ (both set to the same value), see Figure 1. The 2D-potential was calculated on a $5^{\circ}$ grid along the butterfly coordinate and on a $10^{\circ}$ grid along the methyl torsional coordinate. At each point the methyl torsional and butterfly coordinates were kept fixed (three dihedrals in total), all other parameters being optimized.

Figure 9(c) shows the 2D-potential, which exhibits an asymmetric double-minimum shape along the butterfly coordinate with a low barrier of $\sim 400 \mathrm{~cm}^{-1}$. Figures 9 (a) and 9(b) show the two ring-puckered minima, which are separated by $\sim 80 \mathrm{~cm}^{-1}$. They have different equilibrium methyl torsion angles, therefore interconversion between them involves motion along both coordinates. The two minima also have slightly different $V_{3}$ barriers, as listed in Table $\mathrm{V}$. To elucidate the contribution of these two vibrations to the spectrum, we would need to solve the two-dimensional problem. Kleiner and Hougen have proposed an approach for solving 2D-coupled motions in systems with a methyl rotor and a double-minimum oscillatory motion such as ring inversion. ${ }^{66}$ Whereas their approach is optimal for eigenvalues far below the barrier, ${ }^{66}$ we need eigenvalues also above the butterfly barrier of $\sim 400 \mathrm{~cm}^{-1}$. The problem is additionally complicated by the additional involvement of the $\mathrm{NH}_{2}$ inversion mode because besides the up-down and up-up minima in Figure 9 there are also the down-up and down-down minima.

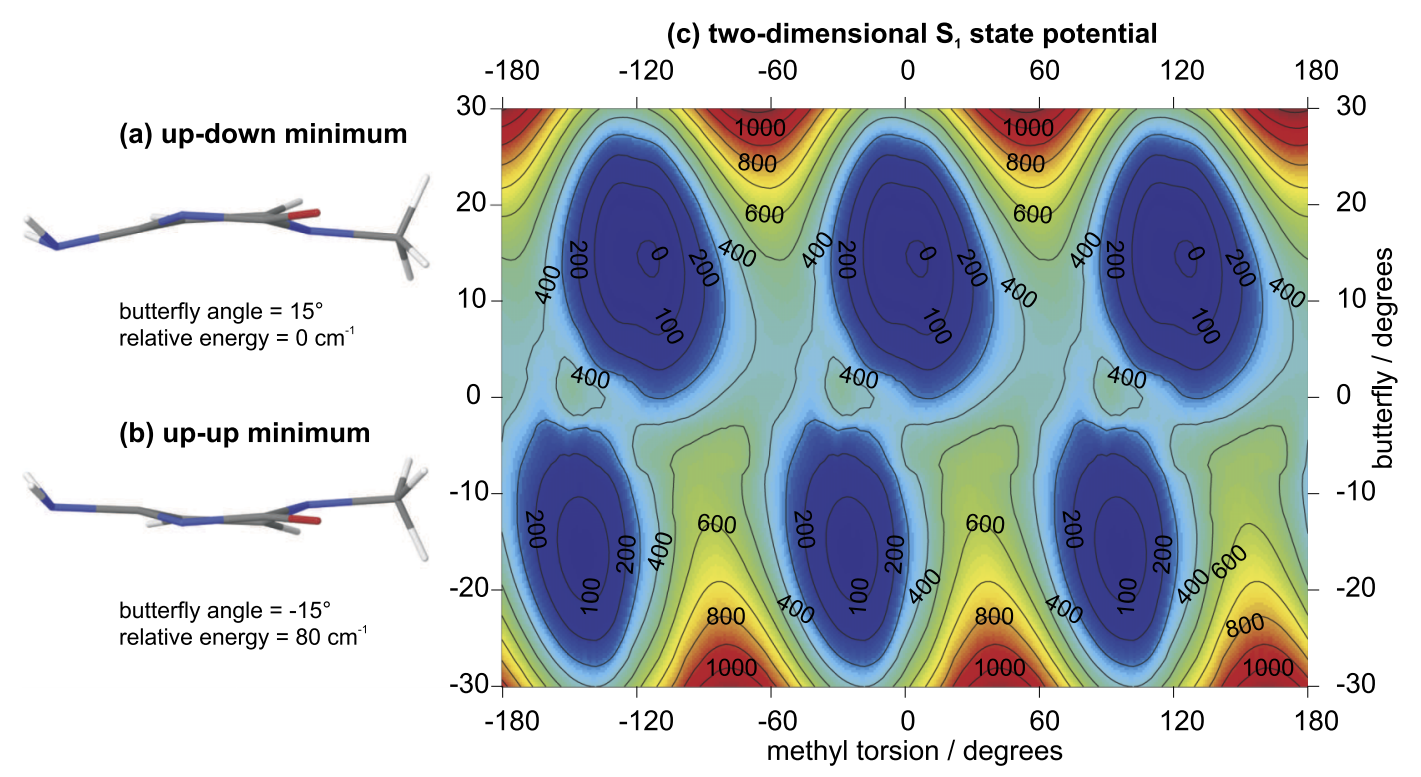

FIG. 9. (a) The up-down and (b) up-up minimum structures of 1-methylcytosine. (c) SCS-CC2/aug-cc-pVDZ calculated $S_{1}$ state two-dimensional potential energy surface along the methyl torsional $(x)$ and butterfly vibrational $(y)$ coordinates, contours are spaced by $100 \mathrm{~cm}^{-1}$. The $x$ axis is given relative to the global minimum shown in (a). The $y$ axis represents the value of the $\mathrm{N}^{3}-\mathrm{C}^{2}-\mathrm{N}^{1}-\mathrm{C}^{6}$ and $\mathrm{C}^{6}-\mathrm{C}^{5}-\mathrm{C}^{4}-\mathrm{N}^{3}$ angles. 


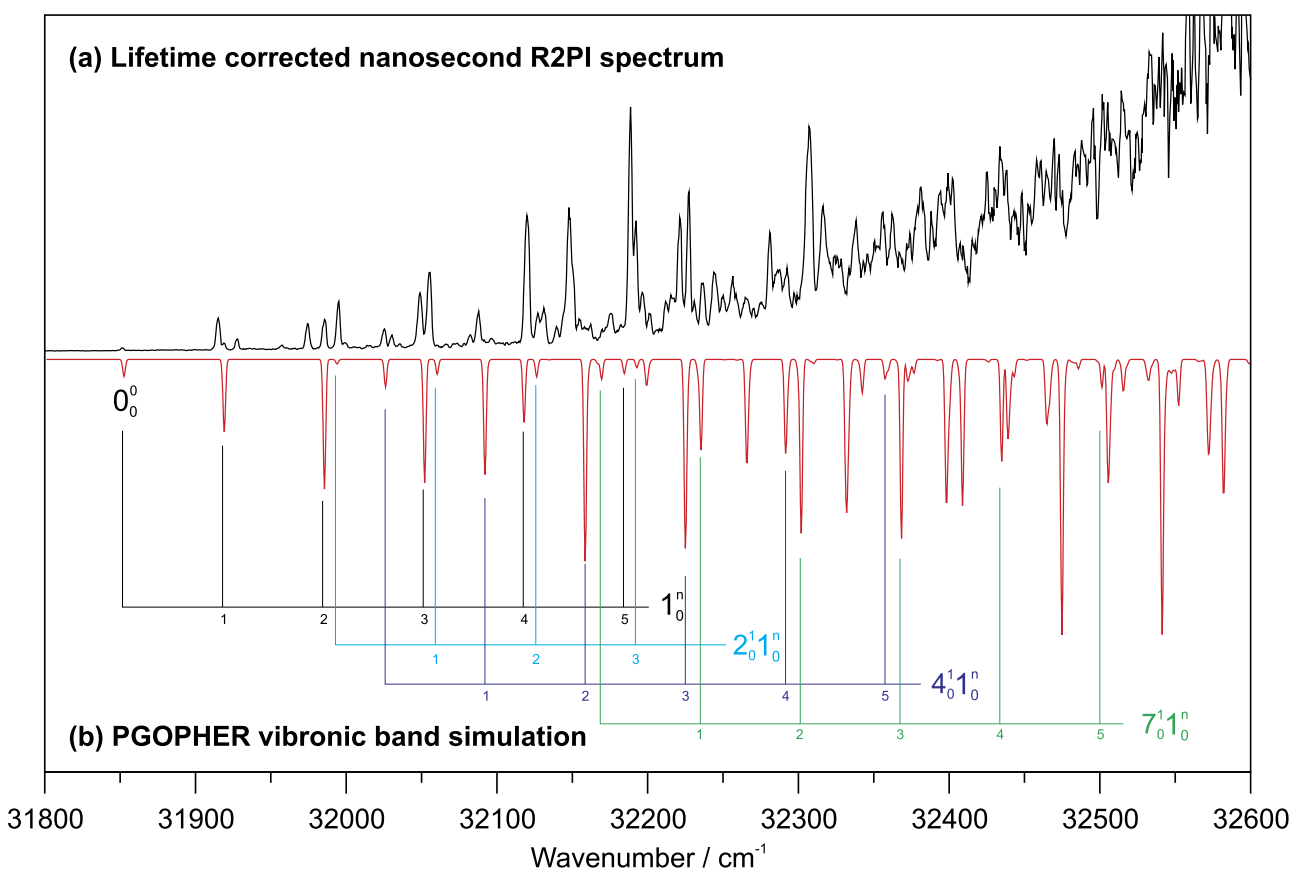

FIG. 10. (a) Lifetime corrected nanosecond resonant two-photon ionization spectrum of 1MCyt, see also Fig. 8 and (b) simulated vibronic spectrum (with PGOPHER, plotted in the negative direction) based on the SCS-CC2/aug-cc-pVDZ ground and ${ }^{1} \pi \pi^{*}$ state calculations.

\section{G. Vibronic assignments}

As pointed out in Section III B, the $S_{1}$ minimum of 1MCyt, $\left(\pi \pi^{*}\right)_{\text {Min }}$, shows strong ring-puckering with the methyl group rotated relative to the $S_{0}$ minimum at the $\operatorname{CASSCF}(12,11) / 6$ 311G**, TD-CAM-B3LYP/6-311G**, and SCS-CC2/aug-cc$\mathrm{pVDZ}$ levels of theory. These large displacements suggest that long progressions should be observed involving at least one out-of-plane mode and the methyl torsion. The PGOPHER simulated vibronic band spectrum of 1MCyt using the SCSCC2/aug-cc-pVDZ optimized $S_{0}$ and $S_{1}$ state equilibrium structures and harmonic wavenumbers is shown in Figure 10. To achieve better agreement with the experimental spectrum, the calculated wavenumber of the butterfly $\left(v_{1}^{\prime}\right)$ mode was decreased from $81.3 \mathrm{~cm}^{-1}$ to $66.5 \mathrm{~cm}^{-1}$ and that of $v_{4}^{\prime}$ from $190.5 \mathrm{~cm}^{-1}$ to $173.3 \mathrm{~cm}^{-1}$; the out-of-plane $v_{2}^{\prime}, v_{5}^{\prime}, v_{7}^{\prime}$, and $v_{9}^{\prime}$ vibrational wavenumbers were included without changes. The PGOPHER simulated vibronic spectrum predicts a long progression in the $v_{1}^{\prime}$ butterfly mode, as well as analogous progressions of $v_{1}^{\prime}$ in combination with the fundamentals of the other excited-state vibrations. The latter exhibit small Franck-Condon factors. Note that the methyl rotor transitions cannot be predicted with PGOPHER. Table VI summarizes the band assignments, which are tentative, especially as regards the methyl rotor transitions.

\section{H. Rotational band contours}

In the $S_{0}$ state, $1 \mathrm{MCyt}$ is an effectively planar asymmetric rotor: the $a$ and $b$ inertial axes lie in the pyrimidinone plane, as shown in Figure 11, and the $c$-axis is perpendicular to this plane.

Figure 12 shows the rotational band contours of several low-lying vibronic bands of $1 \mathrm{MCyt}$ and $1-d_{3}$-MCyt. Due to vibronic band overlap above $0_{0}^{0}+190 \mathrm{~cm}^{-1}$, the rotational contours can no longer be uniquely separated. The contours in Figure 12 exhibit a double-wing shape with a central dip that is characteristic for $a / b$-hybrid bands, which implies that the vibronic transitions are polarized in-plane. This confirms the computational predictions that the $S_{0} \rightarrow S_{1}$ state is a $\pi \pi^{*}$ transition. The $0_{0}^{0}$ and $0_{0}^{0}+64 \mathrm{~cm}^{-1}$ bands of $1 \mathrm{MCyt}$ show an additional sharp peak just below the central dip between the wings. This peak is less pronounced in the contours of the $0_{0}^{0}+123 \mathrm{~cm}^{-1}$ and $0_{0}^{0}+134 \mathrm{~cm}^{-1}$ bands, which indicates a

TABLE VI. $S_{1}$ vibronic frequencies (in $\mathrm{cm}^{-1}$ ) of 1-methylcytosine and assignments. $^{\mathrm{a}}$

\begin{tabular}{|c|c|c|}
\hline Assignment & Frequency & Rel.intensity ${ }^{\mathrm{b}}$ \\
\hline \multirow[t]{2}{*}{$0_{0}^{0}$} & $(31852.2)$ & 1.0 \\
\hline & 64 & 10.8 \\
\hline \multirow[t]{3}{*}{$1_{0}^{1}$ (butterfly) + methyl rotor } & 67 & 2.5 \\
\hline & 75 & 3.8 \\
\hline & 105 & 2.0 \\
\hline \multirow[t]{2}{*}{$1_{0}^{2}+$ methyl rotor } & 123 & 9.0 \\
\hline & 134 & 10.3 \\
\hline $2_{0}^{1}$ & 143 & 16.0 \\
\hline $4_{0}^{1}$ & 173 & 7.3 \\
\hline \multirow[t]{2}{*}{$1_{0}^{3}+$ methyl rotor } & 179 & 5.3 \\
\hline & 197 & 19.0 \\
\hline $2_{0}^{1}+1_{0}^{1}$ & 204 & 25.5 \\
\hline \multirow[t]{2}{*}{$4_{0}^{1}+1_{0}^{1}+$ methyl rotor } & 236 & 13.0 \\
\hline & 268 & 43.0 \\
\hline \multirow[t]{2}{*}{$1_{0}^{4}+$ methyl rotor } & 275 & 13.0 \\
\hline & 279 & 13.1 \\
\hline
\end{tabular}

${ }^{\mathrm{a}}$ Detailed assignments of the $v_{1}^{\prime}$ (butterfly) and methyl-rotor transitions are not possible, since the two modes are coupled, see the text.

${ }^{\mathrm{b}}$ Relative to the $0_{0}^{0}$ transition. 


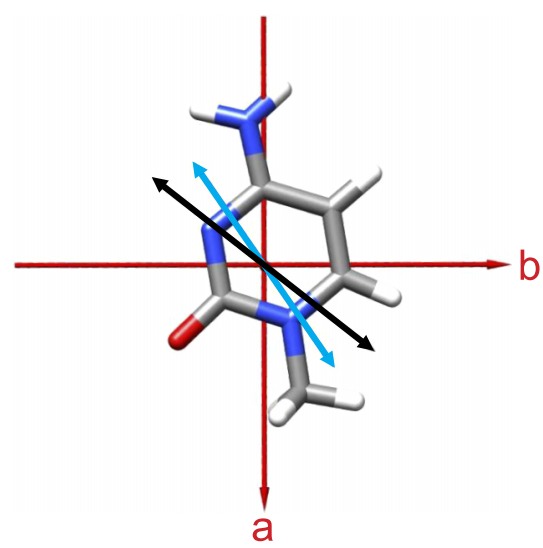

FIG. 11. SCS-CC2/aug-cc-pVDZ calculated (light blue) and experimental (black) transition dipole moment (TDM) vectors of the $S_{0} \rightarrow S_{1}\left({ }^{1} \pi \pi^{*}\right)$ transition of 1-MCyt. The TDM calculation was done at the $S_{0}$ minimum structure. The in-plane $a$ and $b$ inertial axes are drawn in red.

small $c$-type (out-of-plane) contribution to the transition dipole moment (TDM). In fact, while the MS-CASPT2 transition dipole moment along the $c$-axis is zero at the FC geometry, it has a clear non-zero component at the $\left(\pi \pi^{*}\right)_{\text {Min }}$ geometry, which we take as a further indication that the $S_{1}$ minimum is non-planar.

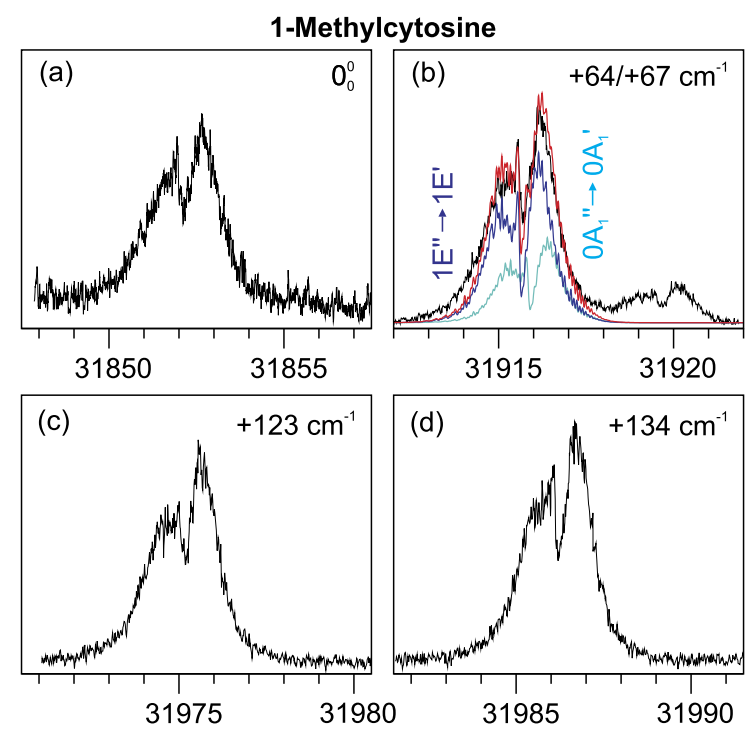

1-( $\left(d_{3}\right)-$ Methylcytosine

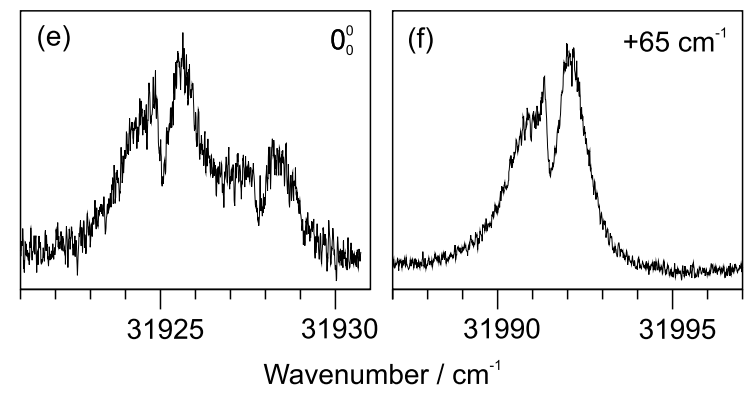

FIG. 12. Two-color R2PI rotational band contours (in black) of supersonically cooled (a)-(d) 1 MCyt and (e) and (f) 1-( $\left.d_{3}\right)$-MCyt with ionization at $223 \mathrm{~nm}$. For the $0_{0}^{0}+64 \mathrm{~cm}^{-1}$ band the simulated contour is shown in red. The simulated contour exhibits a $1 E^{\prime \prime} \rightarrow 1 E^{\prime}$ (blue) and a $0 A_{1}^{\prime \prime} \rightarrow 0 A_{1}^{\prime}$ (cyan) transition, separated by $0.25 \mathrm{~cm}^{-1}$.
Rotational contour simulations for the $0_{0}^{0}+64 \mathrm{~cm}^{-1}$ band of $1 \mathrm{MCyt}$ were performed using the PGOPHER ${ }^{67}$ program and an asymmetric rigid-rotor Hamiltonian. The SCS-CC2 ground-state $A^{\prime \prime}, B^{\prime \prime}$, and $C^{\prime \prime}$ constants given in Table VII were used as fixed inputs. The SCS-CC2 calculated changes of the rotational constants, $A^{\prime}-A^{\prime \prime}, B^{\prime}-B^{\prime \prime}$, and $C^{\prime}-C^{\prime \prime}$, and the transition dipole moment (TDM) components $\mu_{a}, \mu_{b}$, and $\mu_{c}$ were used to initiate the fitting routine. For the laser lineshape we employed a Gaussian with the measured full width at half-maximum $\Delta_{\text {Gauss }}=1.6 \mathrm{GHz}$, see Section II B. The excited-state lifetime $\tau$ of 1MCyt contributes a Lorentzian line shape of width $\Delta_{\text {Lorentz }}=1 /(2 \pi \tau)$ to every rovibronic line, which has to be convoluted with the laser line width $\Delta_{\text {Gauss }}=1.6 \mathrm{GHz}$. We used the excited-state lifetime of $\tau=450 \mathrm{ps}$, as determined by ps pump/delayed ionization measurements, see Section III E. Since the $S_{0}$ state internalrotation levels are cooled to the lowest $0 A_{1}^{\prime \prime}$ and $1 E^{\prime \prime}$ torsional sub-levels, the vibronic bands should exhibit two torsional sub-bands corresponding to the $0 A_{1}^{\prime \prime} \rightarrow 0 A_{1}^{\prime}$ and $1 E^{\prime \prime} \rightarrow 1 E^{\prime}$ transitions. Assuming that the $0 A_{1}^{\prime \prime} \rightarrow 0 A_{1}^{\prime}$ excitation has only half of the intensity of the $1 E^{\prime \prime} \rightarrow 1 E^{\prime}$ excitation, we find that the two sub-bands cannot be separated by more than $0.25 \mathrm{~cm}^{-1}$; when assuming larger sub-band splittings we were no longer able to model the double-wing shape of the contour. If we assume the SCS-CC 2 calculated $S_{0}$ state barrier $V_{3}^{\prime \prime}=361 \mathrm{~cm}^{-1}$ to be qualitatively correct, then the $S_{1}$ state torsional barrier $V_{3}^{\prime}$ has to be $>150 \mathrm{~cm}^{-1}$; this is in agreement with the lowest barrier calculated on the 2D surface, see Section III F.

Although the rotational constants for the $E$-sub-band differ from the $A$-sub-band because of second-order torsionrotation contributions, ${ }^{68-71}$ the same parameters were used for both sub-bands. With the $0 A_{1}^{\prime \prime} \rightarrow 0 A_{1}^{\prime}$ transition located by $0.25 \mathrm{~cm}^{-1}$ to the blue of the $1 E^{\prime \prime} \rightarrow 1 E^{\prime}$ sub-band, first the rotational constant changes $A^{\prime}-A^{\prime \prime}, B^{\prime}-B^{\prime \prime}$, and $C^{\prime}-C^{\prime \prime}$ and then the TDM components were fitted. Figure 12(b) shows the fitted rotational contour in red, with the A- and E-sub-bands in blue. The fitted parameters are summarized in Table VII. For a good agreement with the experimental contour, the $\mu_{b}$ contribution had to be increased from $40 \%$ to $56 \%$, hence the TDM vector is rotated towards the $b$ inertial axis. However, the slow rise on the left side of the experimental contour could not be simulated.

\section{Radiationless decay mechanisms}

To understand the radiationless decay mechanisms, we have calculated the MS-CASPT2//CASSCF energy profiles for the paths to $(E t h)_{X}$ and $(O P)_{X}$, see also Section II A. The decay through $\left(n_{O}, \pi *\right)_{X}$ has not been considered because it is energetically disfavored for Cyt at the MS-CASPT2 level, ${ }^{24,32}$ and it can be expected that 1-methyl substitution will not change this feature. The path to $(E t h)_{X}$ is shown in Figure 13(a). $(E t h)_{X}$ is similar to the structure optimized at the MS-CASPT2 level in Ref. 36 and to the analogues optimized for cytosine at different levels of theory. ${ }^{21-26,30-32}$ Its relative energy is $31375 \mathrm{~cm}^{-1}$. It is characterized by a twisted $\mathrm{C}_{5}-\mathrm{C}_{6}$ bond, with a bond length of $1.468 \AA$ and a $\mathrm{H}-\mathrm{C}_{5}-\mathrm{C}_{6}-\mathrm{H}$ dihedral angle of $122^{\circ}$. The estimated barrier to $(E t h)_{X}$ is 
TABLE VII. $S_{0}$ and ${ }^{1} \pi \pi^{*}$ state SCS-CC2/aVDZ and B3LYP/TZVP calculated rotational constants and transition dipole moments of $1 \mathrm{MCyt}$ and $1-d_{3}-\mathrm{MCyt}$, compared to fitted values from the contour simulation of the $0_{0}^{0}+64 \mathrm{~cm}^{-1}$ band of $1 \mathrm{MCyt}$.

\begin{tabular}{|c|c|c|c|c|c|}
\hline & \multicolumn{3}{|c|}{$1 \mathrm{MCyt}$} & \multicolumn{2}{|c|}{ 1-( $\left(d_{3}\right)$-Mcyt } \\
\hline & B3LYP & $\mathrm{SCS}-\mathrm{CC} 2$ & $0_{0}^{0}+64 \mathrm{~cm}^{-1}$ & B3LYP & SCS-CC2 \\
\hline A"'(MHz) & 3334.6 & 3276.3 & & 3232.7 & 3175.8 \\
\hline B”(MHz) & 1423.9 & 1398.4 & & 1323.6 & 1300.9 \\
\hline C'”(MHz) & 1004.1 & 986.8 & & 950.3 & 34.3 \\
\hline $\mathrm{A}^{\prime}-\mathrm{A}^{\prime \prime}(\mathrm{MHz})$ & -61.0 & -89.7 & -99.0 & -57.9 & -87.1 \\
\hline B'-B”(MHz) & -10.6 & -14.8 & 18.4 & -10.3 & -14.0 \\
\hline $\mathrm{C}^{\prime}-\mathrm{C}^{\prime}(\mathrm{MHz})$ & -10.3 & -1.4 & -13.0 & -9.9 & -1.3 \\
\hline$\left|\mu_{a}\right|:\left|\mu_{b}\right|:\left|\mu_{c}\right|(\mathrm{abs} .)^{\mathrm{a}}$ & $59: 40: 1$ & $59: 40: 1$ & 44:56:0 & $60: 39: 1$ & 61:39:1 \\
\hline$\left|\mu_{a}\right|:\left|\mu_{b}\right|:\left|\mu_{c}\right|$ (emission) $)^{\mathrm{b}}$ & $46: 52: 2$ & $51: 47: 2$ & & $47: 51: 2$ & $52: 46: 2$ \\
\hline
\end{tabular}

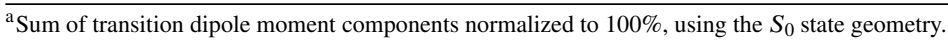

${ }^{\mathrm{b}} \mathrm{Sum}$ of transition dipole moment components normalized to $100 \%$, using the ${ }^{1} \pi \pi{ }^{*}$ state geometry.

$366 \mathrm{~cm}^{-1}$, which is in good agreement with the experimental break-off of vibrational structure observed at approximately $500 \mathrm{~cm}^{-1}$ above the $0_{0}^{0}$ transition. Our estimate is based on an MS-CASPT2 single-point calculation on the CASSCF optimized transition structure and improves previous estimates $\left(1700-3100 \mathrm{~cm}^{-1}\right)$ based on linear interpolations. ${ }^{35,36}$ The
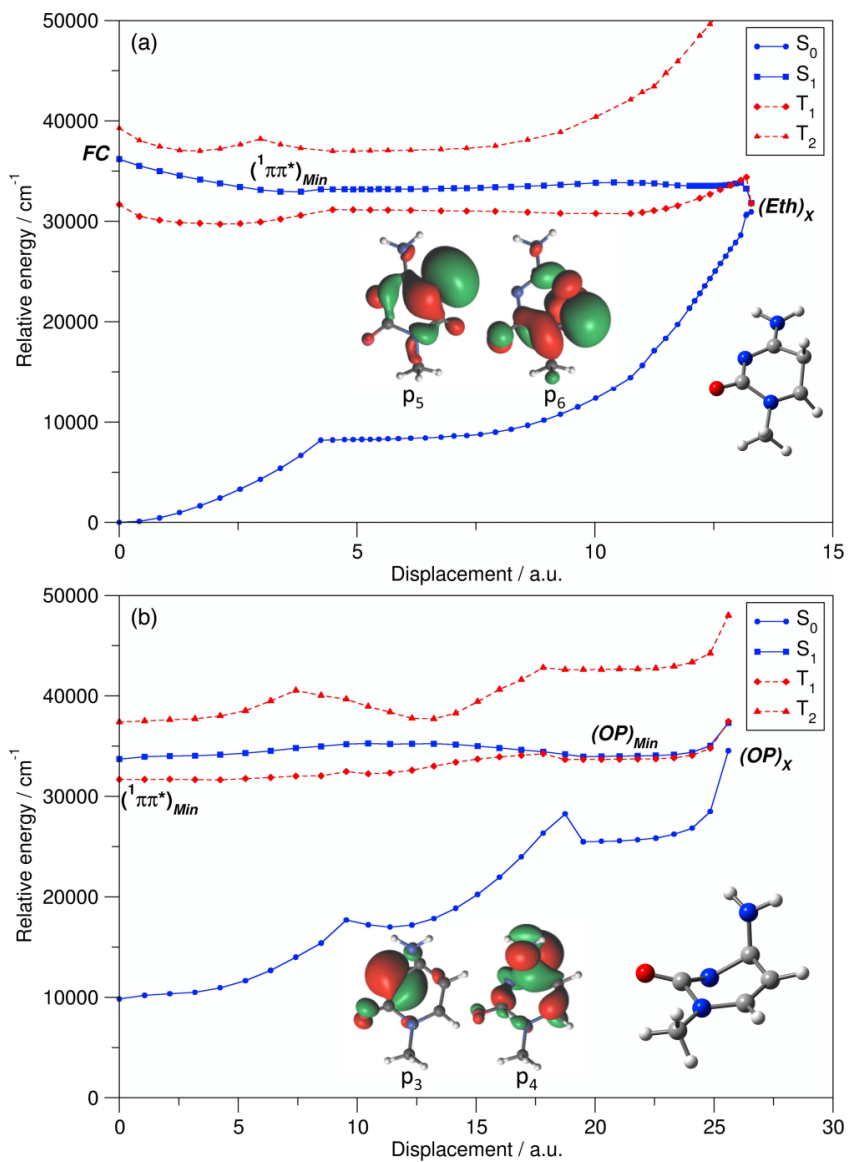

FIG. 13. MS-CASPT2 energy profile of the lowest singlet and triplet states along the CASSCF optimized paths for radiationless decay on $\mathrm{S}_{1}$. (a) From the FC structure to $(E t h)_{X}$ through $\left({ }^{1} \pi \pi^{*}\right)_{M i n}$. (b) From $\left({ }^{1} \pi \pi^{*}\right)_{M i n}$ to $(O P)_{X}$ through $(O P)_{M i n}$. Blue circles: $S_{0}$; blue squares: $S_{1}$; red diamonds: $T_{1}$; red triangles: $T_{2}$. Insets: structure of the $\mathrm{CI}$ and orbitals involved in the excitation. Dominant electronic configurations at the intersections are given in the text. orbitals involved in the excitation at $(\text { Eth })_{X}$ are mainly localized on the $\mathrm{C}_{5}$ and $\mathrm{C}_{6}$ atoms, and the dominant configurations of the intersecting states are $\left(\mathrm{p}_{5}\right)^{2}\left(\mathrm{p}_{6}\right)^{0}$ and $\left(p_{5}\right)^{1}\left(p_{6}\right)^{1}$, see the orbitals in Figure 13(a). In contrast, at $\left({ }^{1} \pi \pi^{*}\right)_{\text {Min }}$ the orbitals involved in the $S_{0} \rightarrow S_{1}$ excitation are delocalized over the conjugated system. This suggests that the small barrier needed to access the CI arises from the change of the wave function along the path, i.e., the localization of the excitation on the $\mathrm{C}_{5}-\mathrm{C}_{6}$ bond.

The alternative decay path goes through $(O P)_{X}$, see Figure 13(b). At this CI, the ring is puckered, with the $\mathrm{N}_{3}$ ring atom and the amino group bent out of the plane $\left(\mathrm{N}_{3}-\mathrm{C}_{4}-\mathrm{C}_{5}-\mathrm{C}_{6}\right.$ and $\mathrm{N}_{8}-\mathrm{C}_{4}-\mathrm{C}_{5}-\mathrm{C}_{6}$ dihedrals of $27^{\circ}$ and $105^{\circ}$, respectively). The decay path from $\left({ }^{1} \pi \pi^{*}\right)_{\operatorname{Min}}$ to $(O P)_{X}$ compares well with the analogous path described for unsubstituted cytosine. ${ }^{18,24-26,30,32}$ It involves a TS with a barrier of $934 \mathrm{~cm}^{-1}$ that leads to another minimum, ${ }^{1}(O P)_{\text {Min }}$, with a relative energy of $242 \mathrm{~cm}^{-1}$ relative to $\left({ }^{1} \pi \pi^{*}\right)_{\operatorname{Min}}$. The $S_{1} / S_{0}$ energy gap at ${ }^{1}(O P)_{\operatorname{Min}}$ is only $8469 \mathrm{~cm}^{-1}$, and the decay path continues to $(O P)_{X}$, which lies at $35972 \mathrm{~cm}^{-1}$. Similar to $(E t h)_{X}$, the excitation is localized on the $\mathrm{N}_{3}-\mathrm{C}_{4}$ bond (orbitals in the inset of Figure 13(b). The configurations of the intersecting states are $\left(\mathrm{p}_{3}\right)^{2}\left(\mathrm{p}_{4}\right)^{0}$ and $\left.\left(\mathrm{p}_{3}\right)^{1}\left(\mathrm{p}_{4}\right)^{1}\right) .(O P)_{X}$ is part of an extended seam ${ }^{72}$ along the $\mathrm{N}_{1}$ pyramidalization coordinate.

\section{J. Triplet state formation and decay}

To explain the formation of the triplet state observed experimentally, we have investigated possible mechanisms for intersystem crossing (ISC) to the triplet state after excitation to the lowest-energy bands of the spectrum. At $\left({ }^{1} \pi \pi^{*}\right)_{M i n}$, the triplet $T_{1}$ and $T_{2}$ states lie approximately $3100 \mathrm{~cm}^{-1}$ below and $5400 \mathrm{~cm}^{-1}$ above $S_{1}$, respectively. $T_{1}$ and $T_{2}$ have $\pi \pi^{*}$ and $n \pi^{*}$ character, respectively, and the calculated spin-orbit coupling (SOC) constants with $S_{1}$ are 2.4 and $10.6 \mathrm{~cm}^{-1}$. This is in agreement with El-Sayed's rules ${ }^{73}$ which predict higher spin-orbit coupling between states with different orbital configurations ( $S_{1}$ and $T_{2}$ in this case). However, ISC to $T_{2}$ is unlikely because the minimum of this state, optimized with TD-CAM-B3LYP/6-311G**, lies $\sim 3100 \mathrm{~cm}^{-1}$ above 
$\left({ }^{1} \pi \pi^{*}\right)_{\text {Min }}$ at the MS-CASPT2 level. In contrast, the $T_{1}$ minimum, which has a semi-planar structure with a small pyramidalization at $\mathrm{C}_{6}$, lies approximately $3100 \mathrm{~cm}^{-1}$ below $\left({ }^{1} \pi \pi^{*}\right)_{\text {Min }}$ (see Table II). Therefore, the most likely mechanism for ISC from $\left({ }^{1} \pi \pi^{*}\right)_{\operatorname{Min}}$ at low excitation energies involves population of the vibrationally excited levels of $T_{1}$, similar to what was recently proposed for Cyt. ${ }^{17}$

We have also calculated the triplet energies along the singlet decay paths (dotted lines in Figures 13(a) and 13(b)). The $S_{1} / T_{1}$ energy gap remains small along both paths, $\leq 3000 \mathrm{~cm}^{-1}$. This suggests that ISC is also possible at higher excitation energies, either along the decay paths to the ground state or by $S_{1} \rightsquigarrow T_{2}$ interconversion. ISC at higher excitation energies has also been observed in mixed quantum-classical dynamics calculations for Cyt. ${ }^{38}$ Finally, decay of $T_{1}$ can take place at a region of degeneracy with $S_{0}$. The optimized $S_{0} / T_{1}$ crossing structure is highly twisted along the $\mathrm{C}_{5}-\mathrm{C}_{6}$ bond $\left(\mathrm{H}-\mathrm{C}_{5}-\mathrm{C}_{5}-\mathrm{H}\right.$ dihedral of $\left.136^{\circ}\right)$ and has an energy of $33500 \mathrm{~cm}^{-1}$, and the calculated SOC element is $2.1 \mathrm{~cm}^{-1}$. The relatively high energy of the crossing explains the long lifetime of the triplet.

\section{CONCLUSION}

Our joint experimental and computational study has allowed us to interpret the R2PI spectrum of 1MCyt and provides detailed insights into the mechanisms of radiationless decay and triplet formation at low excitation energies. The lowest peak of the R2PI spectrum at $31852 \mathrm{~cm}^{-1}$ is assigned as the electronic origin of the spectrum. This band was assigned as a hot band in the previous study of de Vries and co-workers, ${ }^{9}$ but this explanation is excluded by the current UV/UV holeburning experiments. The calculations indicate that the $S_{1}$ minimum has a ring-puckered structure, and the large distortion upon excitation explains the low intensity of the $0_{0}^{0}$ band.

Upon excitation, the ring puckering is coupled with the rotation of the methyl group. This explains the difficulty to simulate the vibronic transitions that are associated with the methyl-rotation mode. For the other modes, the simulated vibronic spectrum predicts a long progression in the $v_{1}^{\prime}$ butterfly vibration plus analogous progressions of $v_{1}^{\prime}$ in combination with the out-of-plane $v_{2}^{\prime}, v_{4}^{\prime}, v_{5}^{\prime}, v_{7}^{\prime}$, and $v_{9}^{\prime}$ excited-state fundamentals, in good agreement with the observed spectrum.

The R2PI spectrum breaks off after approximately $500 \mathrm{~cm}^{-1}$ vibrational excess energy. The calculations give two possible decay paths with low barriers which involve the ethylene and out-of-plane intersections which are well-known from the literature for cytosine. The lowest-energy path leads to the ethylene-type intersection, with a twisted $\mathrm{C}_{5}-\mathrm{C}_{6}$ bond, and has a calculated barrier of $366 \mathrm{~cm}^{-1}$. The alternative path involving puckering of $\mathrm{N}_{3}$ and out-of-plane bending of the amino group has an estimated barrier of $934 \mathrm{~cm}^{-1}$. The good agreement between the experimental and theoretical barrier estimates is a clear indication that, at low vibrational excitation energies, the decay will involve the ethylenic intersection, similar to what has been suggested earlier for Cyt. $^{21-25,31,32}$
The persistence of the R2PI spectrum after 30 ns delayed ionization shows that the triplet state is formed after excitation to the low energy part of the spectrum, and that intersystem crossing to the triplet is the favored decay mechanism at excitation energies below the threshold to the conical intersection. The preferred mechanism under these conditions is ISC to the vibrationally excited levels of $T_{1}$. Overall, the main effect of methyl substitution at $\mathrm{N}_{1}$ on the R2PI spectrum compared to Cyt is a higher density of bands. This is similar to what is found for 5-MCyt. ${ }^{74}$ The break-off of the spectrum is observed at similar excess energies than for Cyt and 5-MCyt, and the mechanism for triplet state formation is similar to the one found for Cyt.

\section{SUPPLEMENTARY MATERIAL}

See the supplementary material for the synthesis of 1-methylcytosine and $d_{3}$-1-methylcytosine, additional computational details, details of UV/UV depletion spectra, delayed ionization spectra, and calculated methyl group internal rotation potentials.

\section{ACKNOWLEDGMENTS}

This research has been supported by the Schweiz. Nationalfonds (Grant Nos. 121993 and 132540), the Agència de Gestió d'Ajuts Universitaris i de Recerca (AGAUR) from Catalonia (Spain) (Grant No. 2014SGR1202), the Ministerio de Economía y Competividad (MINECO) from Spain (Grant No. CTQ2015-69363-P), and the National Natural Science Foundation of China (Grant No. 21303007).

${ }^{1}$ G. Fogarasi, J. Phys. Chem. A 106, 1381 (2002).

${ }^{2}$ S. A. Trygubenko, T. V. Bogdan, M. Rueda, M. Orozco, F. J. Luque, J. Sponer, P. Slavicek, and P. Hobza, Phys. Chem. Chem. Phys. 4, 4192 (2002). ${ }^{3}$ S. Grimme, J. Comput. Chem. 25, 1463 (2004).

${ }^{4}$ Z. Yang and M. T. Rodgers, Phys. Chem. Chem. Phys. 6, 2749 (2004).

${ }^{5}$ J. K. Wolken, C. Yao, F. Turećek, M. J. Polce, and C. Wesdemiotis, Int. J. Mass Spectrom. 267, 30 (2007).

${ }^{6}$ R. Kobayashi, J. Phys. Chem. A 102, 10813 (1998).

${ }^{7}$ O. Kostko, K. Bravaya, A. Krylov, and M. Ahmed, Phys. Chem. Chem. Phys. 12, 2860 (2010).

${ }^{8}$ S. Lobsiger, M. A. Trachsel, H. M. Frey, and S. Leutwyler, J. Phys. Chem. B 117, 6106 (2013).

${ }^{9}$ E. Nir, M. Müller, L. I. Grace, and M. S. de Vries, Chem. Phys. Lett. 355, 59 (2002).

${ }^{10}$ E. Nir, I. Hünig, K. Kleinermanns, and M. S. de Vries, Phys. Chem. Chem. Phys. 5, 4780 (2003).

${ }^{11}$ S. Ullrich, T. Schultz, M. Z. Zgierski, and A. Stolow, Phys. Chem. Chem. Phys. 6, 2796 (2004).

${ }^{12}$ C. Canuel, M. Mons, F. Piuzzi, B. Tardivel, I. Dimicoli, and M. Elhanine, J. Chem. Phys. 122, 074316 (2005).

${ }^{13}$ K. Kosma, C. Schröter, E. Samoylova, I. V. Hertel, and T. Schultz, J. Am. Chem. Soc 131, 16939 (2009).

${ }^{14}$ J. W. Ho, H.-C. Yen, W.-K. Chou, C.-N. Weng, L.-H. Cheng, H.-Q. Shi, S.-H. Lai, and P.-Y. Cheng, J. Phys. Chem. A 115, 8406 (2011).

${ }^{15}$ S. Blaser, M. A. Trachsel, S. Lobsiger, T. Wiedmer, H.-M. Frey, and S. Leutwyler, J. Phys. Chem. Lett. 7, 752 (2016).

${ }^{16}$ S. Lobsiger and S. Leutwyler, J. Phys. Chem. Lett. 3, 3576 (2012).

${ }^{17}$ S. Lobsiger, M. Etinski, S. Blaser, H.-M. Frey, C. Marian, and S. Leutwyler, J. Chem. Phys. 143, 234301 (2015).

${ }^{18}$ N. Ismail, L. Blancafort, M. Olivucci, B. Kohler, and M. Robb, J. Am. Chem. Soc. 124, 6818 (2002).

${ }^{19}$ M. Merchán and L. Serrano-Andrés, J. Am. Chem. Soc. 125, 8108 (2003).

${ }^{20}$ L. Blancafort and M. A. Robb, J. Phys. Chem. A 108, 10609 (2004).

${ }^{21}$ K. Tomic, J. Tatchen, and C. M. Marian, J. Phys. Chem. A 109, 8410 (2005). 
${ }^{22}$ M. Z. Zgierski, S. Patchkovskii, T. Fujiwara, and E. C. Lim, J. Chem. Phys. 123, 081101 (2005).

${ }^{23}$ M. Merchán, R. Gonzalez-Luque, T. Climent, L. Serrano-Andrés, E. Rodríguez, M. Reguero, and D. Peláez, J. Phys. Chem. B 110, 26471 (2006).

${ }^{24}$ L. Blancafort, J. Photochem. Photobiol. 83, 603 (2007).

${ }^{25}$ K. A. Kistler and S. Matsika, J. Phys. Chem. A 111, 2650 (2007).

${ }^{26}$ H. R. Hudock and T. J. Martínez, ChemPhysChem 9, 2486 (2008).

${ }^{27}$ K. A. Kistler and S. Matsika, J. Chem. Phys. 128, 215102 (2008).

${ }^{28}$ L. Serrano-Andrés and M. Merchán, J. Photochem. Photobiol., C 10, 21 (2009).

${ }^{29}$ M. Barbatti, A. J. A. Aquino, J. J. Szymczak, D. NAchtigallova, P. Hobza, and H. Lischka, Proc. Natl. Acad. Sci. U. S. A. 107, 21453 (2010).

${ }^{30}$ M. Barbatti, A. J. A. Aquino, J. J. Szymczak, D. Nachtigallova, and H. Lischka, Phys. Chem. Chem. Phys. 13, 6145 (2011).

${ }^{31}$ M. Kotur, T. C. Weinacht, C. Zhou, K. A. Kistler, and S. Matsika, J. Chem. Phys. 134, 184309 (2011).

${ }^{32}$ A. Nakayama, Y. Harabuchi, S. Yamazaki, and T. Taketsugu, Phys. Chem. Chem. Phys. 15, 12322 (2013).

${ }^{33}$ F. J. Avila Ferrer, F. Santoro, and R. Improta, Comput. Theor. Chem. 1040, 186 (2014)

${ }^{34}$ R. Improta, F. Santoro, and L. Blancafort, Chem. Rev. 116, 3540 (2016)

${ }^{35}$ Q. S. Li and L. Blancafort, Photochem. Photobiol. Sci. 12, 1401 (2013).

${ }^{36}$ A. Nakayama, S. Yamazaki, and T. Taketsugu, J. Phys. Chem. A 118, 9429 (2014).

${ }^{37}$ M. Merchán, L. Serrano-Andrés, M. A. Robb, and L. Blancafort, J. Am. Chem. Soc. 127, 1820 (2005).

${ }^{38}$ M. Richter, P. Marquetand, J. Gonzalez-Vazquez, I. Sola, and L. Gonzalez, J. Phys. Chem. Lett. 3, 3090 (2012).

${ }^{39}$ N. Forsberg and P. A. Malmqvist, Chem. Phys. Lett. 274, 196 (1997).

${ }^{40}$ G. Ghigo, B. O. Roos, and P. A. Malmqvist, Chem. Phys. Lett. 396, 142 (2004).

${ }^{41}$ C. González and H. B. Schlegel, J. Phys. Chem. 94, 5523 (1990).

${ }^{42}$ P. Celani, M. A. Robb, M. Garavelli, F. Bernardi, and M. Olivucci, Chem. Phys. Lett. 243, 1 (1995).

${ }^{43}$ S. Ruiz-Barragan, M. A. Robb, and L. Blancafort, J. Chem. Theory Comput. 9, 1433 (2013).

${ }^{44}$ TURBOMOLE V6.4 2013, A Development of Universität Karlsruhe (TH) and Forschungszentrum Karlsruhe GmbH, 1989-2007, TURBOMOLE $\mathrm{GmbH}$, available from http://www.turbomole.com, accessed 19 May 2015.

${ }^{45}$ The thresholds for scf and one-electron density convergence were set to $10^{-9}$ a.u. and $10^{-8}$ a.u., respectively. The convergence thresholds for all structure optimizations were set to $10^{-8}$ a.u. for the energy change, $6 \cdot 10^{-6}$ a.u. for the maximum displacement element, $10^{-6}$ a.u. for the maximum gradient element, $4 \cdot 10^{-6}$ a.u. for the rms displacement, and $10^{-6}$ a.u. for the rms gradient.

${ }^{46}$ M. J. Frisch, G. W. Trucks, H. B. Schlegel, G. E. Scuseria, M. A. Robb, J. R. Cheeseman, G. Scalmani, V. Barone, B. Mennucci, G. A. Petersson et al., GaUsSian 09 Revision A.02, Gaussian, Inc., Wallingford, CT, 2009.

${ }^{47}$ G. Karlstrom, R. Lindh, P. A. Malmqvist, B. O. Roos, U. Ryde, V. Veryazov, P. O. Widmark, M. Cossi, B. Schimmelpfennig, P. Neogrady, and L. Seijo, Comput. Mater. Sci. 28, 222 (2003).
${ }^{48}$ F. Aquilante, L. De Vico, N. Ferré, G. Ghigo, P.-A. Malmqvist, P. Neogrady, T. B. Pedersen, M. Pitonak, M. Reiher, B. O. Roos, L. Serrano-Andrés, M. Urban, V. Veryazov, and R. Lindh, J. Comput. Chem. 31, 224 (2010).

${ }^{49}$ T. J. Kistenmacher, M. Rossi, J. Caradonna, and L. G. Marzilli, Adv. Mol. Relax. Interact. Processes 15, 119 (1979).

${ }^{50}$ S. Lobsiger, H. M. Frey, and S. Leutwyler, Phys. Chem. Chem. Phys. 12, 5032 (2010).

${ }^{51}$ S. Blaser, H.-M. Frey, C. G. Heid, and S. Leutwyler, Chimia 86, 260 (2014).

${ }^{52}$ S. Lobsiger, R. K. Sinha, S. Blaser, H.-M. Frey, and S. Leutwyler, Nat. Chem. 6, 989 (2014).

${ }^{53}$ Interactive Data Language, Version 7.1, ITT Visual Information Solutions, 2009, www.ittvis.com/idl.

${ }^{54}$ K. A. Kistler, F. C. Spano, and S. Matsika, J. Phys. Chem. B 117, 2032 (2013).

${ }^{55}$ R. Abouaf, J. Pommier, H. Dunet, P. Quan, P.-C. Nam, and M. T. Nguyen, J. Chem. Phys. 121, 11668 (2004).

${ }^{56}$ O. Dolgounitcheva, V. G. Zakrzewski, and J. V. Ortiz, J. Phys. Chem. A 107, 822 (2003).

${ }^{57}$ M. Liu, T. Li, F. S. Amegayibor, D. S. Cardoso, Y. Fu, and J. K. Lee, J. Org. Chem. 73, 9283 (2008).

${ }^{58}$ C. Yao, M. L. Cuadrado-Peinado, M. Polášek, and F. Tureček, J. Mass Spectrom. 40, 1417 (2005).

${ }^{59}$ M. Harańczyk, J. Rak, and M. Gutowski, J. Phys. Chem. A 109, 11495 (2005).

${ }^{60}$ E. C. Lim and J. M. H-Hu, J. Chem. Phys. 45, 4742 (1966).

${ }^{61}$ E. C. Lim, J. Phys. Chem. 90, 6770 (1986).

${ }^{62}$ D. M. Close, J. Phys. Chem. A 108, 10376 (2004), http://pubs.acs.org/doi/ pdf/10.1021/jp046660y.

${ }^{63}$ C. Yu, S. Peng, I. Akiyima, J. Lin, and P. R. LeBreton, J. Am. Chem. Soc. 100, 2303 (1978).

${ }^{64}$ D. G. Lister, J. N. Macdonald, and N. L. Owen, Internal Rotation and Inversion - An Introduction to Large Amplitude Motions in Molecules (Academics Press Inc. (London) Ltd, 1978).

${ }^{65}$ R. M. Dimeo, Simple Quantum Visualizations Using IDL (NIST Center for Neutron Research, 2009).

${ }^{66}$ I. Kleiner and J. T. Hougen, J. Phys. Chem. A 119, 10664 (2015).

${ }^{67}$ C. M. Western, PGOPHER 8.0, a Program for Simulating Rotational, Vibrational and Electronic Structure (University of Bristol, 2015), pgopher.chm. bris.ac.uk.

${ }^{68}$ X.-Q. Tan, W. A. Majewski, D. F. Plusquellic, and D. W. Pratt, J. Chem. Phys. 94, 7721 (1991).

${ }^{69}$ L. Alvarez-Valtierra, J. Yi, and D. W. Pratt, J. Phys. Chem. B 110, 19914 (2006).

${ }^{70}$ L. Alvarez-Valtierra, X.-Q. Tan, and D. W. Pratt, J. Phys. Chem. A 111, 12802 (2007).

${ }^{71}$ P. J. Morgan, L. Alvarez-Valtierra, and D. W. Pratt, J. Phys. Chem. A 113, 13221 (2009).

${ }^{72}$ L. Blancafort, ChemPhysChem 15, 3166 (2014).

${ }^{73}$ M. El-Sayed, Acc. Chem. Res. 1, 8 (1968).

${ }^{74}$ M. A. Trachsel, S. Lobsiger, and S. Leutwyler, J. Phys. Chem. B 116, 11081 (2012). 